\title{
Status of research toward the ITER disruption mitigation system
}

\author{
E. M. Hollmann, ${ }^{1}$ P. B. Aleynikov, ${ }^{2}$ T. Fülöp, ${ }^{3}$ D. A. Humphreys, ${ }^{4}$ V. A. Izzo, ${ }^{1}$ M. Lehnen, ${ }^{2}$ \\ V. E. Lukash, ${ }^{5}$ G. Papp, ${ }^{6}$ G. Pautasso, ${ }^{6}$ F. Saint-Laurent, ${ }^{7}$ and J. A. Snipes ${ }^{2}$ \\ ${ }^{1}$ Center for Energy Research, University of California, San Diego, La Jolla, California 92093-0417, USA \\ ${ }^{2}$ ITER Organization, Route de Vinon sur Verdon, CS 90046 - 13067 Saint Paul lez Durance, France \\ ${ }^{3}$ Department of Applied Physics, Chalmers University of Technology, Göteborg, Sweden \\ ${ }^{4}$ General Atomics, P.O. Box 85608, San Diego, California 92186-5608, USA \\ ${ }^{5}$ Institute of Nuclear Fusion, RRC Kurchatov Institute, Moscow, Russia \\ ${ }^{6}$ Max-Planck Institute for Plasma Physics, EURATOM Association, Garching, Germany \\ ${ }^{7}$ CEA, IRFM, Saint Paul lez Durance, France
}

(Received 10 February 2014; accepted 19 March 2014; published online 17 November 2014)

An overview of the present status of research toward the final design of the ITER disruption mitigation system (DMS) is given. The ITER DMS is based on massive injection of impurities, in order to radiate the plasma stored energy and mitigate the potentially damaging effects of disruptions. The design of this system will be extremely challenging due to many physics and engineering constraints such as limitations on port access and the amount and species of injected impurities. Additionally, many physics questions relevant to the design of the ITER disruption mitigation system remain unsolved such as the mechanisms for mixing and assimilation of injected impurities during the rapid shutdown and the mechanisms for the subsequent formation and dissipation of runaway electron current. (C) 2014 AIP Publishing LLC.

[http://dx.doi.org/10.1063/1.4901251]

\section{INTRODUCTION}

The ITER tokamak will need to operate close to its performance boundaries to achieve its goal of fusion gain $Q_{D T}=10$, and occasional disruptions will probably be an unavoidable part of this high performance operation, as performance boundaries are established. To avoid unnecessary delays in ITER operation due to wall material damage, it is important to mitigate the damaging effects of disruptions to the greatest extent possible, beginning at the start of ITER plasma operations. The U.S. has been given the responsibility of designing the ITER disruption mitigation system (DMS), with a final design review to be presented to the ITER Organization for approval in 2017. The U.S. will also be responsible for the DMS hardware to be delivered to ITER. The physics of disruptions, ${ }^{1}$ their prediction, ${ }^{2}$ and their consequences to the vessel wall ${ }^{3}$ have been reviewed previously. Here, we focus on the present status of research in support of the DMS for ITER.

Section II describes the general global requirements of the ITER DMS system, such as current quench (CQ) duration; as well as DMS engineering constraints, such as limitations on the amount of injected impurities. Section III then gives an overview of different methods for impurity injection. Sections IV-VI describe different forms of disruption damage and their mitigation: heat loads, vessel forces, and runaway electrons (REs). Finally, Sec. VII contains a brief summary and discussion.

\section{DMS DESIGN REQUIREMENTS}

The ITER DMS system is constrained by various design requirements. Here, we coarsely attempt to divide these requirements into hard constraints (due to dangers to the
ITER first wall) and soft constraints (due to space availability, administrative requirements, DMS lifetime, etc.)

\section{A. Hard constraints}

An overview of constraints on the ITER DMS has been given previously. ${ }^{4,5}$ The frequency of disruptions is presently assumed to be of order 1 disruption every 20 shots. This frequency and the severity of disruptions will probably vary depending on the phase of operations. For each disruption at full deuterium-tritium (DT) performance, it will be necessary to dissipate $350 \mathrm{MJ}$ of thermal energy and up to $1 \mathrm{GJ}$ of magnetic energy safely in the vessel walls. It is anticipated that most disruptions in ITER will have a warning time of at least $20 \mathrm{~ms}$ (due to the plasma dropping out of $\mathrm{H}$-mode, loss of vertical control, etc.). Therefore, the thermal quench (TQ) onset time (time from DMS trigger to plasma TQ) is desired to be faster than $20 \mathrm{~ms}$, ideally, $10 \mathrm{~ms}$. The TQ duration itself in ITER is expected to be of order $1-2 \mathrm{~ms},{ }^{1}$ so if a disruption begins without precursor, it will not be possible to mitigate the TQ. To avoid melting the tungsten divertor with conducted heat loads, greater than $90 \%$ mitigation of TQ conducted heat loads is desired. Additionally, the TQ radiation should be as uniform as possible to avoid beryllium first wall melting. For example, assuming a $2 \mathrm{~ms}$ TQ duration, a toroidal peaking factor (TPF) and poloidal peaking factor (PPF) of less than about two each are necessary to avoid beryllium melting (where peaking factor has the usual definition of max divided by mean). The CQ duration should be greater than $50 \mathrm{~ms}$ (with $35 \mathrm{~ms}$ as a hard boundary) to avoid damage due to torques on blanket modules and first wall. Additionally, the CQ duration should be shorter than $150 \mathrm{~ms}$, so that there is not enough time for the plasma to drift into the wall, resulting in additional vertical forces and heat loads on the vacuum vessel 


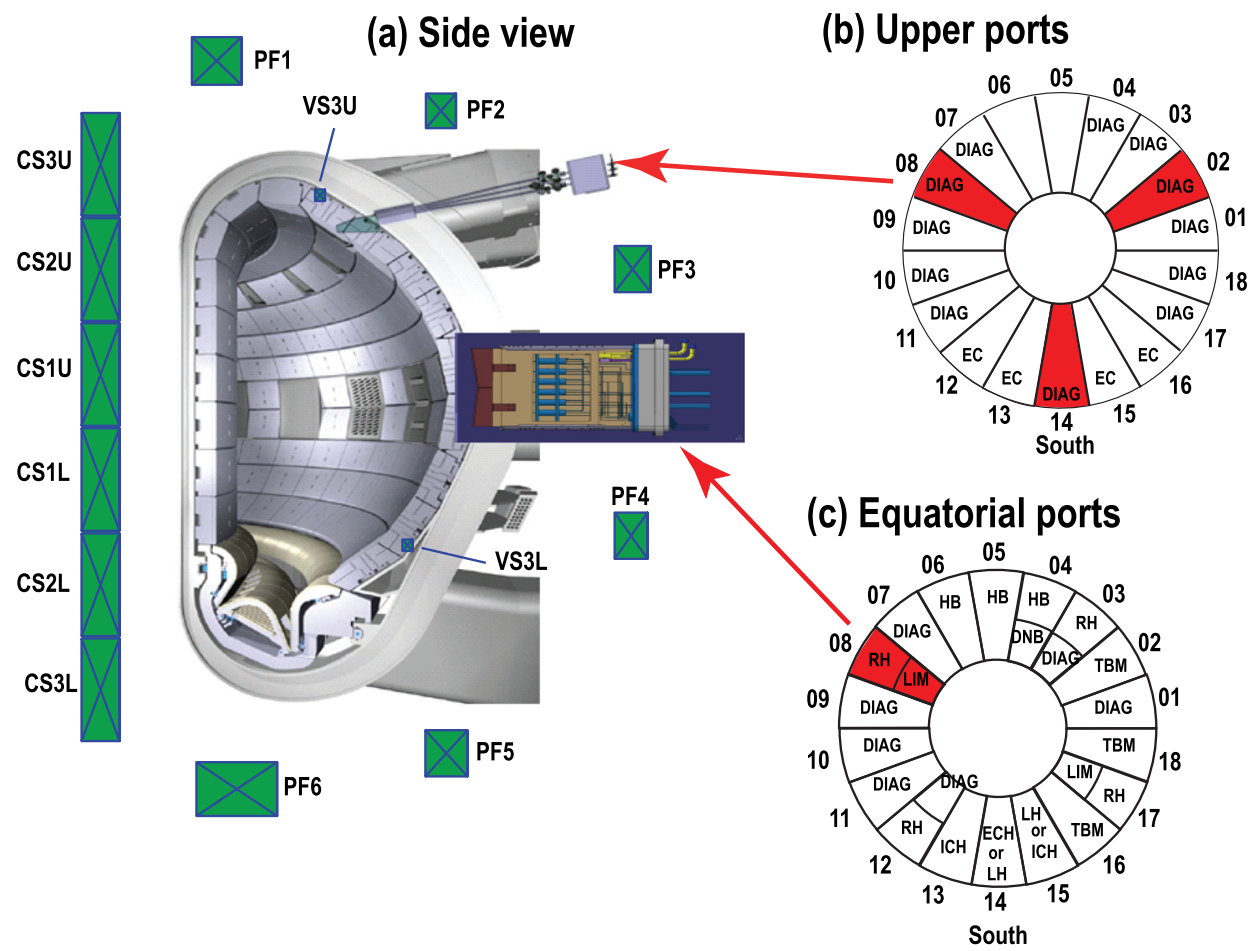

FIG. 1. Overview of port space allocated for ITER DMS system.

due to direct plasma contact. Runaway beam strikes on the ITER wall should have a plasma current of less than 2 MA. RE beam formation times are expected to be of order $20 \mathrm{~ms}$, while RE plateau lifetimes of order $100 \mathrm{~ms}$ or more, so DMS timing requirements for RE plateau mitigation are less stringent than for TQ mitigation or for RE beam formation (CQ avalanche) mitigation.

\section{B. Soft constraints}

The ITER DMS system is constrained by available port space. Presently, parts of four ports are allocated for the DMS system: three upper ports, spaced toroidally by $120^{\circ}$, and a single equatorial port. This layout is shown in Fig. 1. The three upper port plugs (UPPs) have VIS/IR imaging as their main priority, with DMS given only the lower $10 \%$ of each port plug front area. On the equatorial port plug (EPP), the DMS is allocated a vertical slice about $30 \mathrm{~cm}$ wide and $2 \mathrm{~m}$ high, corresponding to roughly $1 / 3$ of the front area.

The magnetic field in the port plugs is about $3 \mathrm{~T} \mathrm{DC}$, so this will tend to saturate ferromagnetics, meaning that normal solenoid valves cannot be used here. Administrative gas leak rate requirements into the vacuum chamber are very strict, $10^{-9} \mathrm{~Pa}-\mathrm{m}^{3} / \mathrm{s}$ for weld joints and flanges. The administrative leak rates for the DMS are expected to be less strict, but are not yet defined. Maintaining low leak rates will be challenging for high speed gas valves, which must hold off high gas pressures (up to 100 bars) from vacuum.

Port plugs will be removable, but this operation will take several months. The port cell, which is about 4 (or 7) $\mathrm{m}$ away from the vacuum vessel for midplane (or upper) port plugs, on the other hand, can be accessed on a shorter $(\sim 1$ week) cool-down time scale, so routine maintenance could be performed there more easily. Because of the poor port plug accessibility, a 4000 shot (approximately 2 yr) maintenance-free reliability is desired for the DMS in-port- plug components. Power, cooling, and cryogenic lines can be run for the DMS, but the ITER building design is already frozen, so lines will need to conform to existing clearance holes. New holes are not permitted (due to safety issues and the expense of new neutron streaming calculations).

For DMS system reliability, the DMS system design will need to take into account the ITER radiation environment. The radiation environment in port plugs is shielded by a steel and water labyrinth and exact doses will depend on location of valves in port plug and degree of protection by labyrinth, but neutron fluxes can be in the range $10^{13}-10^{17}$ neutrons $/ \mathrm{m}^{2} / \mathrm{s}$ and gamma dose rates in the range $10^{-2}-10^{2} \mathrm{~Gy} / \mathrm{s}$ during plasma shots. These correspond to roughly $10 \times$ higher fluxes than JET and fluences that are many orders of magnitude higher.

In the interest of shot cycle speed, it is desired that system recovery time after a DMS shutdown be less than $3 \mathrm{~h}$. As a consequence, there are limits on the amount of gas that can be injected into the vessel in one shutdown, set by limitations in the tritium processing plant. The injected gas limitations are $40 \mathrm{kPa}-\mathrm{m}^{3}$ of $\mathrm{He}, 50 \mathrm{kPa}-\mathrm{m}^{3}$ of $\mathrm{D}_{2}$, or $100 \mathrm{kPa}-\mathrm{m}^{3}$ of $\mathrm{Ne}$ or $\mathrm{Ar}^{4}$

Beryllium can be injected into the vessel, but cannot damage the chamber walls, ruling out fast solid pellets. Another safety issue is dust formation. $\mathrm{W}$ dust is a radiation hazard in the case of a vessel breach, while hot Be dust is a potential explosion hazard. In-vessel limits are presently $670 \mathrm{~kg}$ (total cold dust), $11 \mathrm{~kg}$ (hot Be dust), and $77 \mathrm{~kg}$ (hot $\mathrm{W}$ dust). ${ }^{6}$ However, the allowable amount of additional $\mathrm{Be}$ which can be intentionally added by each DMS shutdown is much lower, only $15 \mathrm{~g}{ }^{4}$

\section{IMPURITY INJECTION HARDWARE}

Rapid shutdown has been demonstrated in tokamaks by a wide range of mass injection methods: massive gas 
injection (MGI), ${ }^{7}$ small cryogenic pellet injection, ${ }^{8}$ large shattered cryogenic pellet injection (SPI), ${ }^{9}$ large solid pellet injection (LSP), ${ }^{10}$ and laser-blow off. ${ }^{11}$ Additional novel mass injection methods have been proposed such as liquid jets ${ }^{12}$ or plasma jets. ${ }^{13}$ Each method has its own advantages and disadvantages relative to the others. Combination methods, e.g., LSP followed by MGI, have also been proposed, but not investigated experimentally. MGI is relatively straightforward to implement but tends to have a slow impurity delivery ramp-up and poor mixing efficiency into the plasma. ${ }^{14}$ Small cryogenic pellets do not deliver enough radiating material for TQ mitigation (for low-Z pellets) and tend to create large runaway electron seeds (for high-Z pellets). ${ }^{10}$ Large shattered pellets are challenging to design and optimize. Large solid pellets can damage the vessel walls and need to be carefully designed to have good impurity deposition in the plasma core. Laser blow-off is difficult to re-load in a tokamak environment and tends to inject insufficiently small amounts of impurities. Liquid and plasma jets can achieve potentially very high impurity delivery velocities but are challenging to build and have not been tested in the tokamak environment. At the moment, the mass injection techniques favored for rapid shutdown in ITER appear to be MGI, SPI, and (possibly) LSP; these techniques are elaborated below. Visible camera images of impurity radiation resulting from different rapid shutdown methods are shown in Fig. 2; the main point here is to illustrate the different impurity deposition profiles of the different rapid shutdown methods.

\section{A. Gas injection hardware}

Most MGI experiments to date have been done with fast valves. The dynamics of flow resulting from gas valves opening into vacuum has been studied in detail both experimentally ${ }^{15}$ and theoretically. ${ }^{16}$ The flow of gas into an evacuated tube is in the "friction limited regime" for small tube diameters, quickly transitioning into the "valvelimited" regime if the tube diameter is larger than the valve diameter. Most present MGI experiments are in the valvelimited regime. The front of the gas pulse thus arrives on a time scale $L / 3 c_{s}$ and then rises to steady flow on a time scale $L / c_{s}$, where $L$ is the tube length and $c_{s}$ is the gas sound speed. Finite valve opening time will broaden the flow characteristic. When the valve is turned off, the mass flow rate decays on a time scale $L / c_{s}$. Pressure sag in the gas reservoir will lower the mass flow rate; this becomes a consideration for MGI valve designs with small reservoir volume.

Present MGI gas valves typically have opening and closing times of order $1 \mathrm{~ms}$, orifice sizes of order $1 \mathrm{~cm}$ or less, and maximum fill pressures of order 50 bars. Designing reliable valves significantly larger or faster than this appears challenging. For example, moving to much larger orifice sizes can lead to sealing and chattering issues. Valve chattering (pre-pulses) need to be avoided, as these could cause weak early gas delivery and a poorly mitigated TQ with high heat conducted heat loads.
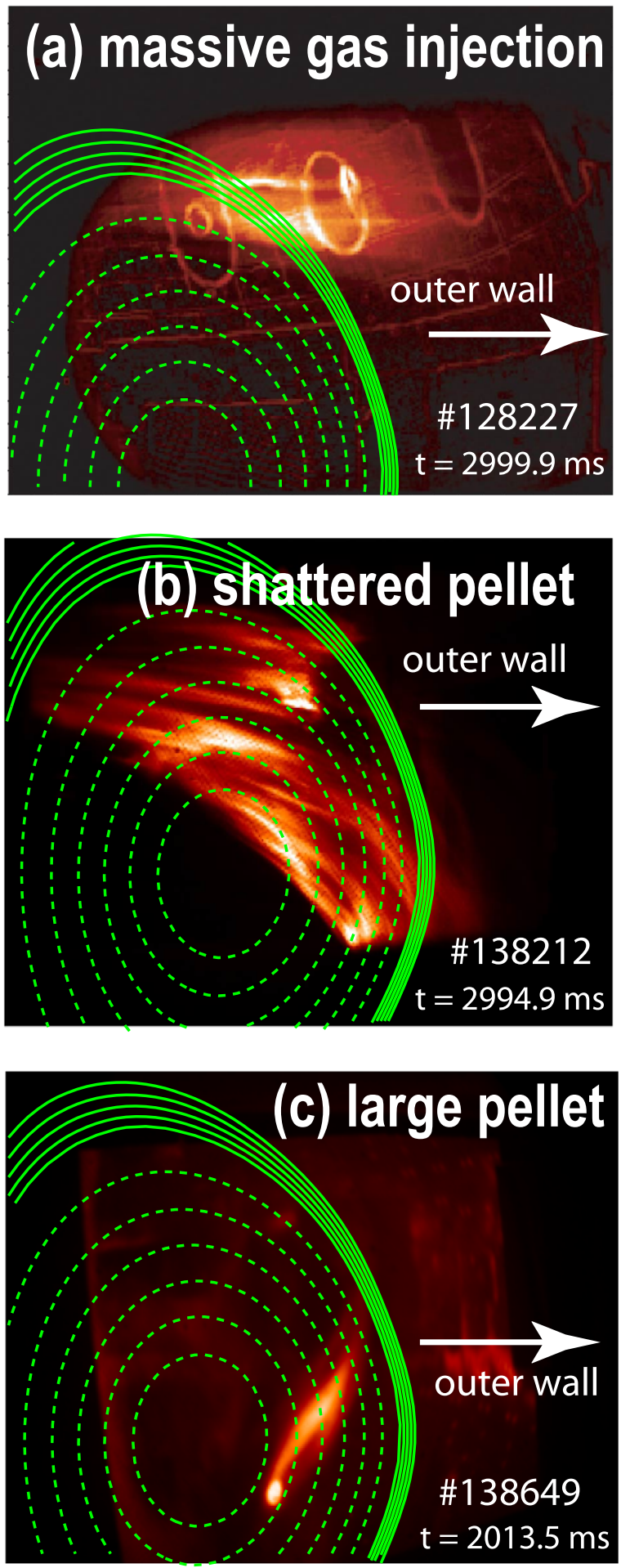

FIG. 2. Examples of visible images from different rapid shutdown methods from DIII-D showing (a) massive gas injection, (b) shattered pellet injection, and (c) large intact pellet injection. Green curves are approximate plasma magnetic flux surface contours in tangency plane for orientation only.

A variety of different MGI valve designs have been tested in present tokamaks including fast solenoid valves, ${ }^{17}$ two-stage solenoid/pressure driven valves, ${ }^{18}$ two-stage piezo valve/spring driven valves, ${ }^{19}$ and eddy-current driven valves. ${ }^{15}$ The eddy-current design appears favored for use in the ITER port plugs, as it is only weakly affected by the strong DC magnetic fields (although there is some torque on 
the valve due to the current in the valve coil and the DC magnetic fields). In all cases, degradation of the MGI valve seals due to the radiation environment needs to be considered.

An example of rapid shutdown timescales resulting from fast valve MGI shutdown in DIII-D is shown in Figs. 3(a)-3(f). It can be seen that there is a significant delay $(>10 \mathrm{~ms})$ between the initial jet trigger and the TQ. MGI shutdown timescales have been shown to depend on many parameters within each tokamak including gas species, plasma thermal energy, plasma $q$-profile, and distance between valve and plasma edge. Despite these many dependencies, rough overall increasing trends with machine size in MGI shutdown timescales can be seen when looking at different machines; this is illustrated in Figs. 3(g) and 3(h). The dashed lines in Figs. 3(g) and 3(h) are simple linear fits to the data. The dashed curve in Fig. 3(i) is a quadratic fit, since CQ duration is expected to increase as minor radius squared. The JET data does not seem to follow this trend well; it is not certain if this is real or an artifact of the small number of data points used here. Also, the reason for the apparently reduced cold front duration on MAST when compared with other devices, Fig. $3(\mathrm{~g})$, is not understood; one speculative possibility may be that this is a result of the low aspect ratio of MAST.

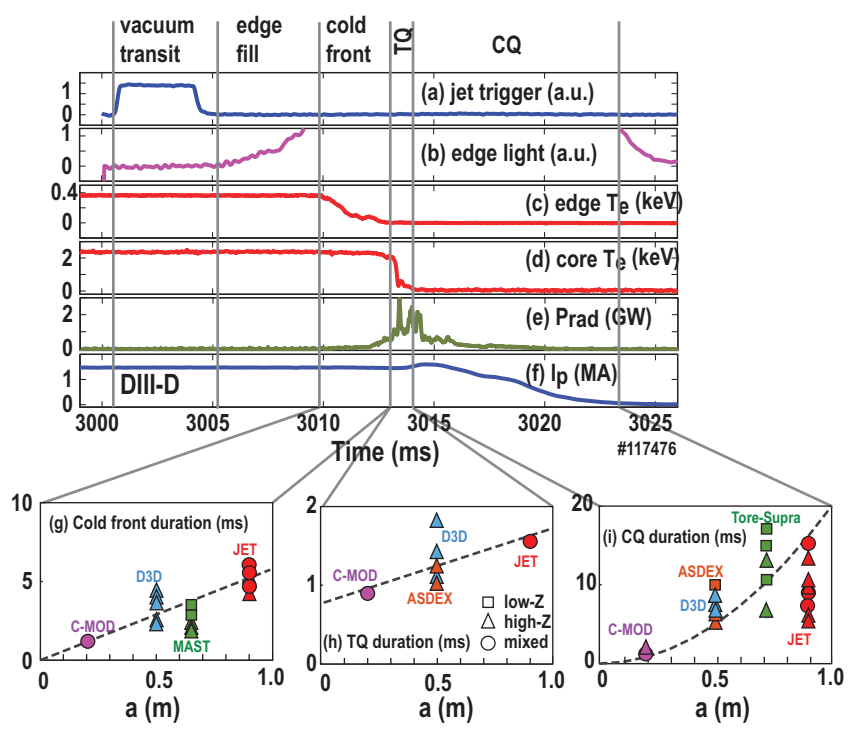

FIG. 3. (a)-(f) Example of rapid shutdown time traces for MGI shutdown in DIII-D, as well as (g)-(i) overview of minor radius (machine size) trends in rapid shutdown timescales in some present tokamaks. CQ durations in (i) are $90 \%-10 \%$ times and are taken from measurements using coils internal to the conducting wall. Reproduced by permission of IAEA, Vienna from E. M. Hollmann et al., Nucl. Fusion 48, 115007 (2008). Copyright 2008 IOP Publishing; R. S. Granetz et al., Nucl. Fusion 47, 1086 (2007). Copyright 2007 IOP Publishing; C. Reux et al., Nucl. Fusion 50, 095006 (2010). Copyright 2010 IOP Publishing; A. J. Thornton et al., Nucl. Fusion 52, 063018 (2012). Copyright 2012 IOP Publishing; M. Lehnen et al., Nucl. Fusion 51, 123010 (2011). Copyright 2011 IOP Publishing; G. Pautasso et al., Nucl. Fusion 51, 103009 (2011). Copyright 2011 IOP Publishing; E. M. Hollmann et al., Nucl. Fusion 45, 1046 (2005). ${ }^{92}$ Copyright 2005 IOP Publishing; and M. L. Reinke et al., Nucl. Fusion 48, 125004 (2008). ${ }^{93}$ Copyright 2008 IOP Publishing. Reproduced by permission from A. J. Thornton et al., Plasma Phys. Controlled Fusion 54, 125007 (2012). Copyright 2012 IOP Publishing.
An alternate to using a fast valve is MGI using a rupture disk,$^{20}$ which has been demonstrated in Tore-Supra. ${ }^{21}$ In this technique, the gas is pre-filled into high-pressure cartridges placed close to the plasma. The cartridge rupture disk can be triggered by various methods; in the Tore-Supra experiments, a brief multi-kA arc was run through a filament inside the cartridge. Very high pressures (90-150 bars) were successfully sealed in the cartridges and very short $(0.3 \mathrm{~ms})$ valve opening times were achieved. Due to the fast valve opening time, fast flow rise time, and proximity to the plasma, very rapid ( $2 \mathrm{~ms}$ ) shut down (trigger to TQ) times were achieved, about $4 \times$ faster than with comparablespecies MGI in Tore-Supra. Rupture disks are being considered for use in the ITER DMS, but various technical issues remain to be studied, including the effect of neutrons on the rupture disk reliability, avoiding the possibility of high-Z rupture disk leaf metal being fired into the plasma,${ }^{18}$ and reloading the high-pressure cartridges after use.

\section{B. Pellet injection hardware}

Typically, pellets are gas-accelerated by roomtemperature low- $Z$ gas; the resulting pellet velocities are found to be well described by the ideal gas gun formula, ${ }^{22}$ reaching velocities of around $300-600 \mathrm{~m} / \mathrm{s}$, depending on the tube length, pellet mass, and propellant gas. These velocities are comparable to typical gas sound speeds, so the arrival time of large pellets at the plasma is not significantly different from MGI impurities launched from the same location. The deposition characteristics of pellets are very different from MGI, however, because pellets penetrate into the plasma farther than gas jets and the impurities are deposited more suddenly. These differences in deposition characteristics can be both beneficial, e.g., assimilation of more radiating impurities can result in more complete mitigation of conducted heat loads; or detrimental, e.g., the sudden deposition of impurities at one toroidal location could result in unacceptably high radiated main chamber heat loads at that location.

Another disadvantage of pellets is that they can damage wall tiles: for example, if they arrive too late and are not sufficiently ablated by the cold CQ plasma. The SPI concept aims to address this issue - the pellet is pre-broken on a shatter plate into shards before entering the plasma. The SPI concept can therefore give a very rapid pulse of impurity deposition without endangering wall tiles. As an additional possible advantage, impurity ablation is expected to be faster for the shattered pellets when compared with a single large pellet and over a larger area, giving a larger radiation-heated wall surface area. A variety of SPI breaker plate geometries have been bench tested at ORNL, including a double breaker plate, v-groove, single bend, and double-bend tubes. ${ }^{23}$ Controlling the composition of the shattered pellet is challenging - invariably, some distribution of particles, liquid, and gas results from the pellet breakup, and this appears to be more or less fixed by the pellet velocity and breaker plate geometry. ${ }^{18}$

Large solid (non-cryogenic) pellets (LSPs) may be attractive for ITER because they do not put any load on the tritium processing plant, potentially reducing post-shutdown 
recovery time. Beryllium is the favored material, as it is already present in the machine; although, in principle, other low-Z solids such as lithium boron, or beryllium hydride could be used as well. The principal concern with LSPs is the possibility of damage to machine tiles from the pellet impact. Several methods of addressing this, such as hollow pellets, porous pellets, and shell pellets, have been proposed. Shell pellets are attractive in theory, as the pellet payload could be optimized in advance: for example, a beryllium shell filled with beryllium powder of a desired size mixed with high pressure argon of the desired pressure could be used. Some preliminary tests of shell pellets have been done on DIII-D. ${ }^{10}$ The pellets had a diameter of $1 \mathrm{~cm}$ and were made of polystyrene shells filled with boron powder. Optimizing the shell thickness was found to be challenging: shells which were too thick were found to pass completely through the plasma without breaking open, while shells that were too thin could break during acceleration or going around guide tube bends.

\section{DMS SHUTDOWN HEAT LOADS}

\section{A. Characterization}

Heat flow during the TQ is not well understood, but based on extrapolation from present machines, ITER is expected to have a central $T_{e}$ collapse (TQ) time of about $1-2 \mathrm{~ms}$, and a resulting heat deposition time scale on the wall of about $3 \mathrm{~ms}$. Together with an expected TPF of about 4, unacceptably high heat loads on the lower strike points and also on the upper Xpoint region of ITER are predicted. ${ }^{24}$ Typically, unmitigated disruption strike point broadening in ITER is expected to be of order 7; this is based on measurements on a variety of tokamaks (JET, AUG, DIII-D, MAST, etc.), which measure of order $5-10 \times$ strike point broadening during disruptions. ${ }^{25}$

Significant wall material sputtering occurs as a result of TQ heat loads and plasma flux. This plays an important role in subsequent disruption dynamics as highlighted by large $(3 \times)$ increase in CQ duration seen in JET when changing from carbon to beryllium main wall. ${ }^{26}$ It is possible that this large effect may be due to a reduced Be sputtering rate during disruptions, as may result from reduced Be dust and flake production when compared with carbon. Evidence for this is a $5 \times$ decrease in dust signal during normal operation with the beryllium first wall, when compared with carbon. ${ }^{26}$ Beryllium and carbon also have somewhat different radiation characteristics, which could also serve to explain the JET results. ${ }^{27}$

In addition to wall material sputtering, hydrogen (and/or deuterium) is released from walls during disruptions. The amount of hydrogen released in carbon wall experiments appears to be of order the initial particle content in the plasma ${ }^{28,29}$ and can thus affect CQ duration ${ }^{30}$ and RE formation. ${ }^{31}$ In JET, beryllium wall hydrogen release appears about $2 \times$ less than carbon release, and is therefore still not negligible. ${ }^{26}$

\section{B. Mitigation}

Heat loads during DMS shutdown will come from a combination of radiation and conduction. Predicting the resulting heat loads to the wall is a complex coupled problem depending on DMS impurity deposition, plasma impurity transport, and plasma response (MHD and heat transport). For MGI impurity deposition in plasma, experiments in a variety of tokamaks are in agreement that the injected impurities are stopped at the edge of hot pre-TQ plasmas, even when the jet ram pressure is greater than the plasma pressure, indicating that the jet ablation pressure and/or toroidal magnetic field pressure are contributing to jet stopping. ${ }^{32}$ MGI impurity assimilation during the CQ is more difficult to measure, partially due to the slow toroidal transit time of impurities during the CQ and the lack of good CQ diagnostics. It is expected that direct penetration of gas should be easier during the CQ (due to lower ablation pressure), but plasma mixing of impurities should also be lower (due to lower thermal energy). It is clear from line-integrated electron density measurements and fast camera imaging that some assimilation of injected impurities does occur during the CQ. Overall, disruption-averaged fueling efficiencies from MGI appear to be of order 5\%-20\% for high-Z gases and higher (up to $50 \%$ ) for low-Z gases like helium, ${ }^{14,35}$ with TQ assimilation typically appearing to be better than CQ assimilation.

For pellet impurity deposition in plasma, the ablation of both cryogenic and solid (refractory) pellets has been studied extensively in theory. ${ }^{33,34}$ Experimentally, pellet ablation rates appear well described by theory for small (less perturbative) pellets. However, for large pellets, ablation rates appear lower than predicted by theory, perhaps due to anomalous fast cross-field heat transport. ${ }^{10}$ During the $C Q$, pellets in DIII-D are typically observed to pass directly through the plasma without ablating significantly; however, this is for a carbon-wall machine with very low CQ electron temperature and may not apply to CQ plasmas with higher $T_{e}$.

Plasma parallel expansion of deposited ions during MGI shutdown is rapid: for example, parallel flow timescales measured in ASDEX Upgrade (AUG) are about $0.5-1 \mathrm{~ms}^{36}$ DIII-D measured similar numbers, about $1-2 \mathrm{~ms},{ }^{14}$ consistent with fairly cold $\left(T_{e} \approx 5 \mathrm{eV}\right)$ impurity ions. Initial inward impurity ion diffusion appears slower: $\left(D_{\perp} \approx 1 \mathrm{~m}^{2} / \mathrm{s}\right)$ initially, then accelerating (perhaps $10 \times$ ) during the TQ. There are also indications of accelerated parallel flow during the TQ. In pellet shutdown experiments, there is some evidence for decreased MHD and possibly decreased TQ parallel mixing when compared with MGI shutdown. It is not known at this time to what degree these observations will scale to ITER, e.g., if radial impurity mixing also increase by perhaps $10 \times$ during the TQ of ITER.

Poloidal flows appear to play a role in many MGI shutdowns, with flows from the outer midplane over the top of the machine and down to the center post observed during the shutdown in fast bolometry in various tokamaks; ${ }^{14,36,37}$ examples are shown in Fig. 4. This flow often appears to have a filamentary structure. The origin of these flows is not understood yet. Experiments reversing toroidal magnetic field were not able to find a clear (e.g., $E \times B$ flow with positively charged plasma) explanation for the flow direction in vertical displacement event disruptions. ${ }^{38}$ Data on poloidal flow velocities are limited, but the poloidal mixing appears to occur on a roughly $2 \mathrm{~ms}$ time scale in DIII-D, AUG, and on a somewhat slower $(\sim 5 \mathrm{~ms})$ time scale on JET. These 
(a) $t=61.753 \mathrm{~s}$

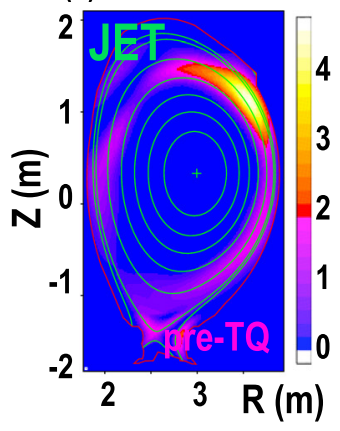

(b) $t=61.757$

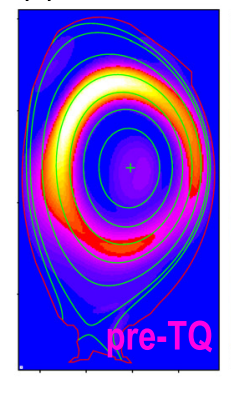

(e) $\mathrm{t}=2000.8 \mathrm{~ms}$

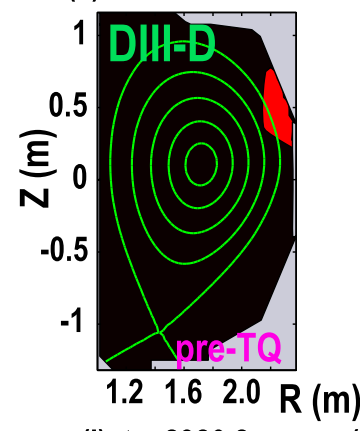

(f) $\mathrm{t}=\mathbf{2 0 0 1 . 3}$

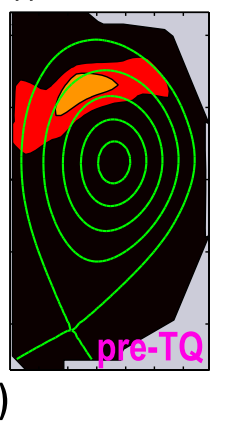

(g) $t=2002.1$
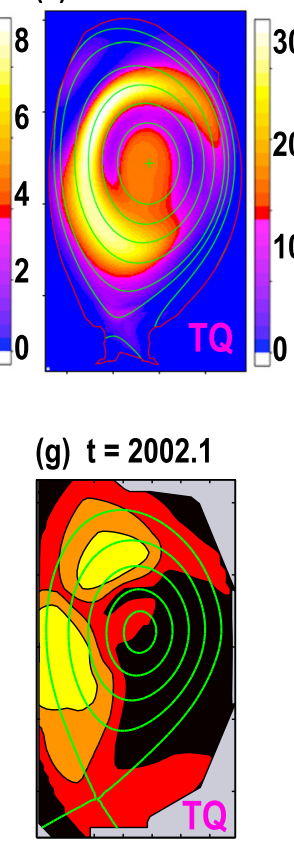

(d) $\mathrm{t}=61.762$
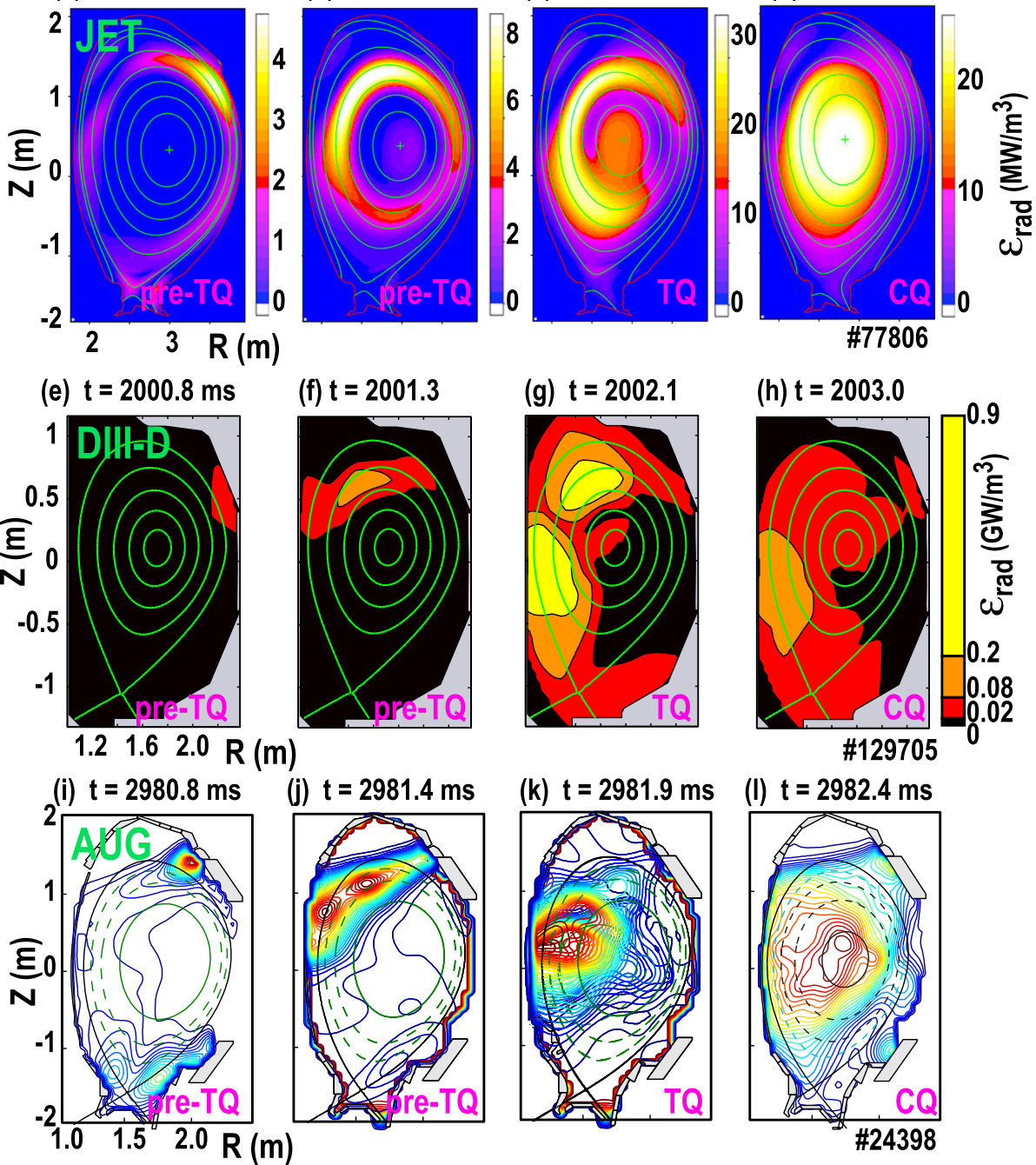

(k) $\mathrm{t}=2981.9 \mathrm{~ms}$

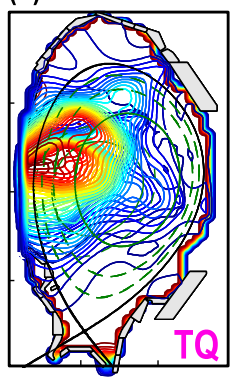

(h) $t=2003.0$

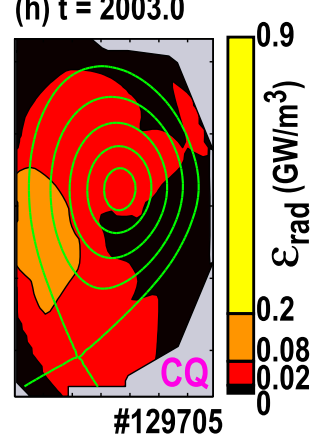

(I) $\mathrm{t}=\mathbf{2 9 8 2 . 4 \mathrm { ms }}$

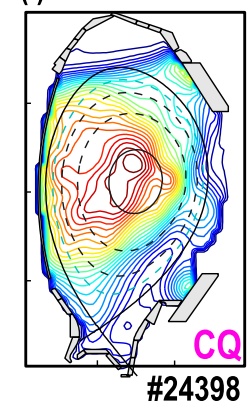

FIG. 4. Poloidal flows measured by fast bolometry during MGI shutdowns showing (a)-(d) $90 \% \mathrm{D}_{2} / 10 \% \mathrm{Ar}$ MGI shutdown in JET; (e)-(h) Ne MGI shutdown in DIII-D; and (i)-(1) $\mathrm{He}$ MGI shutdown in $\mathrm{AUG}^{36}$ (with color scale varying frame-frame). Reproduced by permission of IAEA, Vienna from E. M. Hollmann et al., Nucl. Fusion 48, 115007 (2008). Copyright 2008 Elsevier. Reprinted with permission from A. Huber et al., "Radiation loads onto plasma-facing components of JET during transient events-experimental results and implications for ITER,” J. Nucl. Mater. 415, S821-S827 (2011). Copyright 2011 Elsevier. flows could be beneficial in the sense that they act to poloidally distribute radiating impurities, thus spreading radiated heat loads. However, the flows could be detrimental if they are strong enough to result in impurities piling up on the center post and radiating preferentially there.

Similar to the particle transport discussed above, crossfield plasma heat transport appears to be relatively slow initially in MGI shutdown experiments, $\chi_{\perp} \approx 1 \mathrm{~m}^{2} / \mathrm{s}$, then accelerating rapidly (perhaps $10 \times$ ) during the TQ MHD. Experiments have seen indications that heat transport can be slightly faster than particle transport, i.e., that a cold front moves in faster than the impurity front following particle injection into the plasma edge. Cold front propagation has been seen to be affected by rational surfaces. ${ }^{39}$ The importance of the $q=2$ rational surface in the TQ onset has been demonstrated in several MGI experiments, ${ }^{39-41}$ but there are suggestions that the $q=2$ surface appears to play a less important role during pellet injection experiments. During the $\mathrm{CQ}$, there is very little data available on heat transport and impurity transport. Impurity mixing during the CQ appears to be slower than the TQ. However, significant structure and motion is observed in camera images, indicating that some mixing is still occurring.
MGI shutdowns on a variety of tokamaks are in agreement that at least small quantities (roughly of order $1 \%$ or more for rapid MGI shutdowns) of high- $Z$ impurities are necessary to avoid significant (50\% or greater) divertor heat loads during the TQ. ${ }^{48,49,57}$ With sufficient high-Z impurity injection, most tokamaks, with sufficiently rapid impurity delivery, report being able to radiate $90 \%+$ of the initial thermal energy during the TQ. Global conducted heat load measurements are hard to achieve due to camera viewing access and fast time scale issues. Additionally, IR emission from the injected gas can confuse camera images. Overall, radiated power data indicate that radiated energy fractions over the whole disruption can exceed $90 \%$ for rapid high-Z gas injection. However, conducted heat load mitigation needs to be greater than $\sim 95 \%$ to avoid divertor melting during the TQ in ITER. This number depends on the conducted heat load wetted area, which is not well known, although Tore-Supra has measured limiter heat load broadening of order $10 \times$ during MGI shutdowns, suggesting a strike point broadening similar to that in unmitigated disruptions. ${ }^{43}$ Evidence for significant conducted heat loads during the CQ has been observed in MAST $^{44}$ and this area has received renewed interest with the recent observations of long 

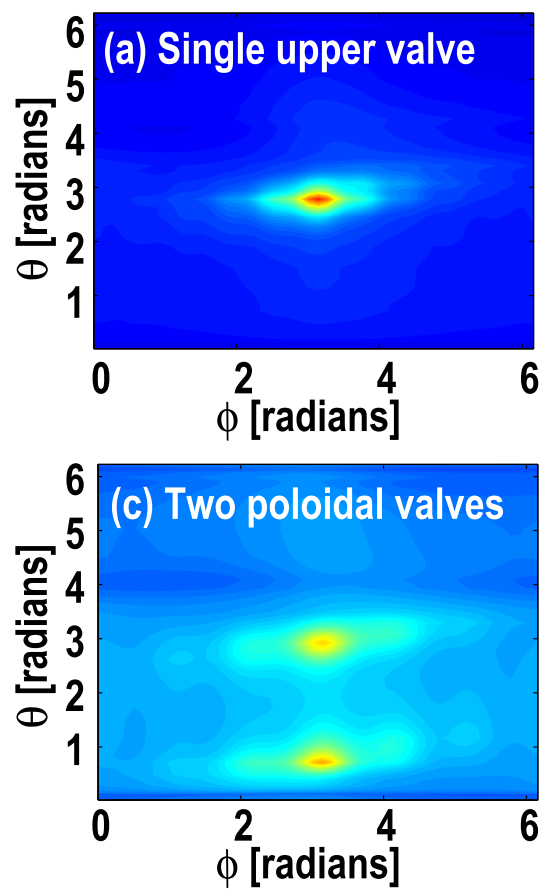

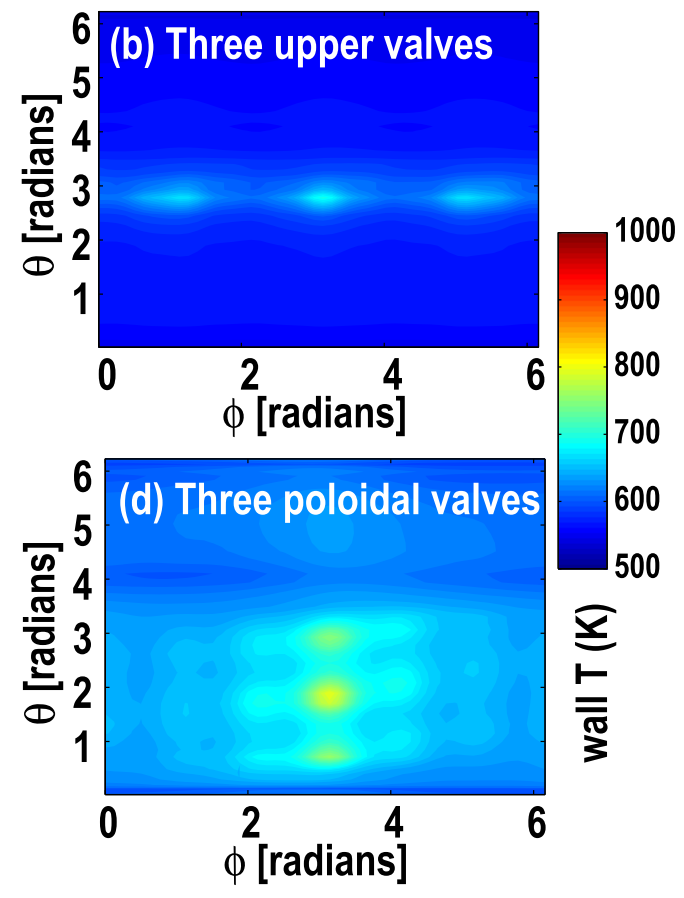

FIG. 5. 2+1D (TokSys) simulations of ITER wall temperature contours during start of TQ for Ne MGI for (a) a single valve, (b) three toroidally space valves, (c) two poloidally spaced valves, and (d) three poloidally spaced valves. In each case, $40 \mathrm{kPa}-\mathrm{m}^{3}$ of $\mathrm{Ne}$ is injected in a $20 \mathrm{~ms}$ pulse.
(100 ms) CQ times (and high CQ temperatures) in JET with the new Be first wall.

Some effort has been put into discussing atomic physics relevant to radiation in ITER TQ plasmas. Due to the large electron densities $\left(n_{e} \approx 10^{20} / \mathrm{m}^{3}\right)$, there is expected to be some reduction in radiation from coronal radiation rates. Additionally, due to the large spatial scales of the impurities $(\sim 1 \mathrm{~m})$ opacity is expected to play a role, at least for resonance lines; this has the beneficial effect of extending the CQ duration and reducing RE avalanching. ${ }^{45}$

The role of valve location on heat load mitigation has only begun to be investigated. C-MOD has done experiments with two toroidally separated valves. ${ }^{42}$ A clear reduction in peak pre-TQ radiated heat loads was seen, but no clear reduction in peak TQ radiated heat loads was observed when using two valves fired simultaneously. AUG has done MGI shutdown experiments with poloidally separated valves-experiments have been done both with an outer upper midplane MGI shutdown and with an inner wall MGI shutdown. Inner wall injection was found to provide significantly $(\sim 3 \times)$ faster shutdown, indicating faster mixing/assimilation from this location. However, this improvement was found to decrease with increasing injection rates, with the inner wall/outer wall injection difference disappearing at large injection rates. ${ }^{19}$

Some simulations have been done of MGI shutdown heat load mitigation. In NIMROD simulations, it was found that there is an intrinsic lower limit on TQ heat load uniformity of order TPF $\approx 2$, set by the strong TQ MHD $n=1$ mode causing toroidally localized radial heat flux. ${ }^{46}$ These results seem consistent with the C-MOD measurements, which find a significant TPF in radiated power, even with two toroidally spaced MGI valves. The NIMROD simulations indicated that the rotating lower order MHD modes could lock to an external perturbation (even including the MGI gas jet!) causing increased heat loads at certain toroidal locations. These simulations did not include plasma rotation, which could affect these results. The NIMROD simulations also predicted much stronger assimilation for outer midplane MGI (vs out of midplane MGI), due stronger plasma gradients in the outer midplane. TokSys simulations using a diffusive approximation for perpendicular heat and particle transport ${ }^{47}$ found that some reduction in peak radiated wall temperature was obtained by using three toroidally or poloidally spaced valves, as shown in Figs. 5 and 6.

As mentioned earlier, a $<20 \mathrm{~ms}$ "trigger to TQ" delay is desired for the ITER DMS. Assuming a $7 \mathrm{~m}$ MGI delivery tube, "first light" time scales are around 4-7 ms (depending on species) and subsequent ramp up times to full flow are around $10-25 \mathrm{~ms}$, resulting in anticipated "trigger to TQ" delays of order $30 \mathrm{~ms}$ (depending on valve size and flow rate). These considerations seem to favor putting MGI valves in the port plugs, where $<20 \mathrm{~ms}$ TQ onset time should be achievable given sufficient valve size and flow rate. Mixedspecies gas jets could be useful to speed delivery of higher-Z gas to the plasma. ${ }^{48,49}$ However, simulations indicate that mixing species could actually delay TQ onset in some cases, due to the lower concentration of radiating impurities. SPI, which has faster mixing, could greatly reduce the TQ onset time, as shown in Fig. 6(e). This faster deposition by SPI has a potential drawback that there is less time for toroidal mixing giving more toroidally localized radiation and higher wall heating at that toroidal location, as shown in Fig. 6(f).

\section{DMS SHUTDOWN VESSEL FORCES}

\section{A. Characterization}

The engineering margin on unmitigated disruption CQ vessel forces is fairly low in ITER, with an engineering margin of $20 \%$ over largest expected unmitigated disruption forces. It is therefore essential that the ITER DMS system do nothing to increase vessel forces over those of an unmitigated disruption. As mentioned in Sec. II A, CQ times which 
Effect of MGI valve location
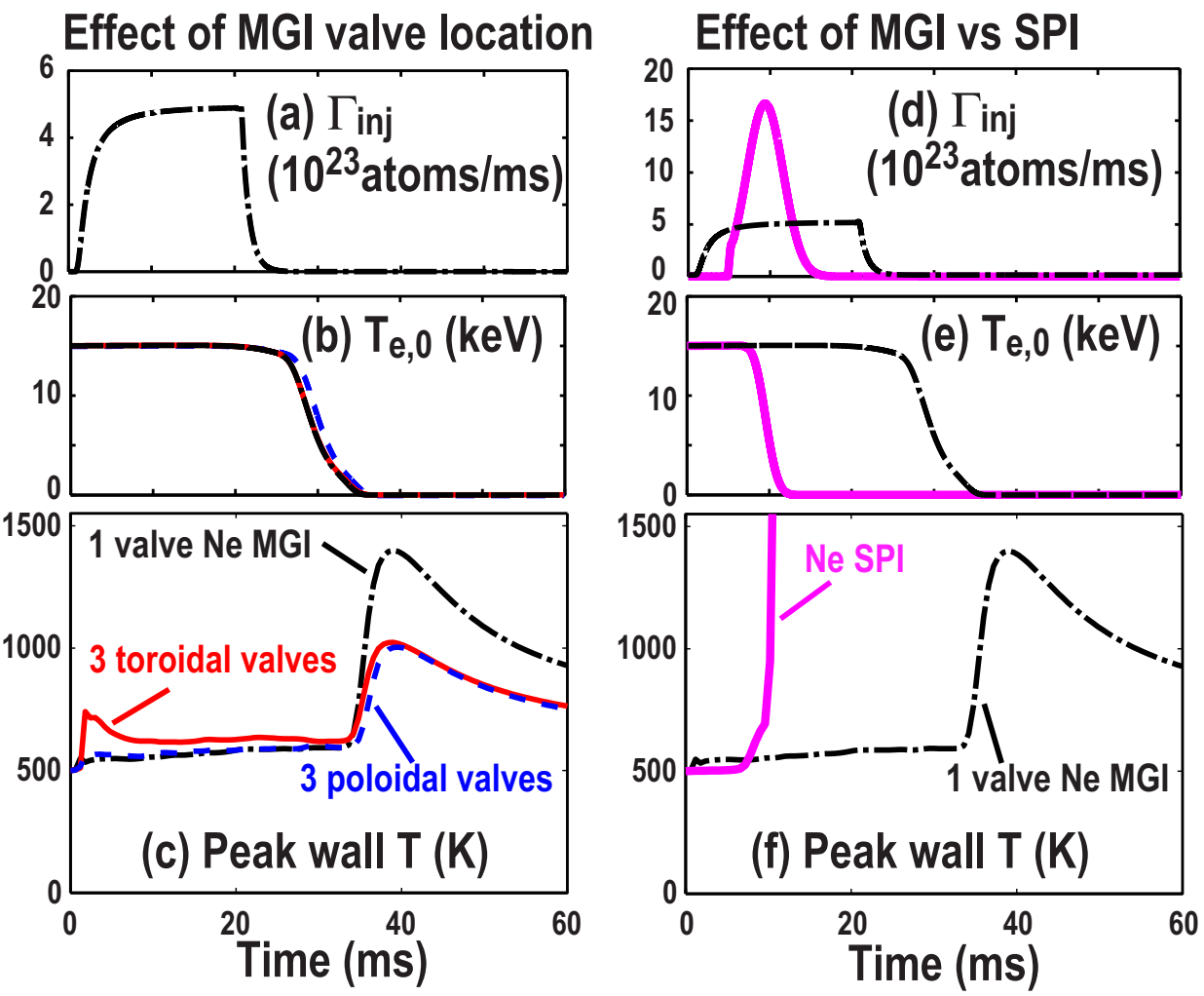

FIG. 6. 2+1D (TokSys) simulations showing time traces of ITER rapid shutdowns for: (a)-(c) Ne MGI for different injection geometries and (d)-(f) Ne SPI compared with Ne MGI. Time traces show impurity delivery rate (a) and (d), central electron temperature (b) and (e), and peak wall temperature (c) and (f) due to radiation only. $40 \mathrm{kPa}-\mathrm{m}^{3}$ of $\mathrm{Ne}$ are delivered in each case using a $2 \mathrm{~m}$ tube. For MGI, a $20 \mathrm{~ms}$ pulse length $100 \mathrm{~atm}$ reservoir pressure and $3 \mathrm{~cm}$ valve diameter are used. For SPI, a pellet velocity of $400 \mathrm{~m} / \mathrm{s}$ and Gaussian deposition profile vs. minor radius is assumed. Perpendicular transport is assumed to be $D_{\perp}=\chi_{\perp}=2 \mathrm{~m}^{2} / \mathrm{s}$ (pre-TQ) and $D_{\perp}=\chi_{\perp}=10 \mathrm{~m}^{2} / \mathrm{s}(\mathrm{TQ})$.

are shorter than $50 \mathrm{~ms}$ lead to undesirably high induced currents and corresponding eddy current forces on shielding blanket modules, while CQ times of less than $35 \mathrm{~ms}$ are to be avoided completely to limit loads on blanket modules.

Conversely, CQ times of longer than $150 \mathrm{~ms}$ could result in unacceptably high halo current forces due to the vertical instability and current channel motion which invariably accompanies the CQ. Halo currents often have large toroidal asymmetry associated with a short-lived $n=1$ mode. $^{50}$ The ITER main vacuum vessel resonance is $8 \mathrm{~Hz}$, but there are also many smaller resonances at higher frequencies, e.g., $40 \mathrm{~Hz}$. Halo current rotation may be a concern if there is a large toroidal asymmetry in the halo current force and this force rotates at a frequency which drives a vacuum vessel mode. DIII-D has observed halo currents which rotate at about $200-400 \mathrm{~Hz}$, but frequently lock. ${ }^{51}$ AUG and C-MOD have seen halo current rotation at about $1 \mathrm{kHz}$, while JET has seen halo current rotation over a broad range of frequencies $50-200 \mathrm{~Hz}^{53,54} \mathrm{NSTX}$ has also observed halo current rotation over a broad range of frequencies $0-2 \mathrm{kHz}{ }^{55}$ Because of these fairly fast presently observed halo current rotation frequencies, it is often assumed that halo current rotation will not be a concern in ITER; however, this is not certain, since the physics of halo current rotation and their machine size scaling is not understood yet. Modeling efforts to try to understand the physics of halo current diffusion and rotation are ongoing. 56

\section{B. Mitigation}

MGI shutdowns typically have of order $2 \times$ lower halo currents than unmitigated disruptions. ${ }^{49}$ Additionally, sideways forces have been shown to be reduced by an order of magnitude in JET when using MGI. ${ }^{49}$ Higher-Z MGI appears to be somewhat better at reducing halo currents, ${ }^{32}$ although the optimum mixture for halo current reduction in $\mathrm{C}-\mathrm{MOD}$ appeared to be $10 \%$ high-Z and $90 \%$ low-Z. ${ }^{57}$

The recent results from JET showing long (100 ms) CQ durations with $\mathrm{Be}$ walls ${ }^{26}$ are good news in that they suggest a very long (possibly $>100 \mathrm{~ms}$ ) unmitigated CQ duration in ITER. Starting with a long intrinsic CQ duration, the CQ duration can be brought down in a somewhat predicable fashion with the DMS. In carbon machines, on the other hand, CQ durations are usually found to be relatively fast, pinned down to, e.g., around $5 \mathrm{~ms}$ in DIII-D by the large amount of carbon released from the walls during disruptions. ${ }^{52}$

\section{DMS SHUTDOWN RUNAWAY ELECTRONS}

Research is ongoing to understand and attempt to mitigate REs at every stage of their life: in the seed formation phase, prompt loss phase, amplification, plateau phase, and final loss phase. Below, present understanding of the different phases is outlined and then efforts at mitigating the different phases are described.

\section{A. Runaway electron characterization}

\section{Runaway electron seed formation}

The seed formation and subsequent prompt loss of REs are important because it affects the magnitude of the subsequent RE plateau, giving final ITER RE plateau current estimates ranging from 0 to $12 \mathrm{MA} .{ }^{58}$ Dreicer seeds are thought to dominate for low-Z injected impurities and hot tail seeds to dominate for high-Z injected impurities. RE seeds due to tritium decay and Compton scattering of hard $\mathrm{x}$-rays emitted 
by the activated wall are usually small unless Dreicer and hot tail seeds are strongly suppressed. ${ }^{59}$ Although little studied, the location of RE seeds is important because this can affect the prompt loss of REs and also affect the current profile of the plasma as RE current becomes dominant, which in turn affects feedback control of the RE plateau.

Example simulations of MGI shutdown RE formation in extremely different ITER rapid shutdown experiments are shown in Fig. 7. These simulations were performed with the GO code. ${ }^{59}$ In Fig. 7(a), massive $\mathrm{D}_{2}$ deposition is simulated, resulting in almost no RE current. Figure 7(b) illustrates a mixed species $\mathrm{D}_{2} / \mathrm{Ar}$ injection, in this case resulting in a significant (12 MA) RE plateau dominantly formed by a hot tail seed amplified by avalanching. The very large quantity of $\mathrm{D}_{2}$ added in the simulation of Fig. 7(a) is necessary to achieve a reasonable CQ duration. Smaller quantity $\mathrm{D}_{2}$ injections will result in unacceptably long CQ durations, but still without RE formation. Conversely, the simulation of Fig. 7(b) illustrates that a very small quantity of Ar deposited by MGI suffices to cause a short CQ duration and large RE seed formation. Finally, Fig. 7(c) shows a simulation of rapid beryllium injection-in this case, the RE formation is almost
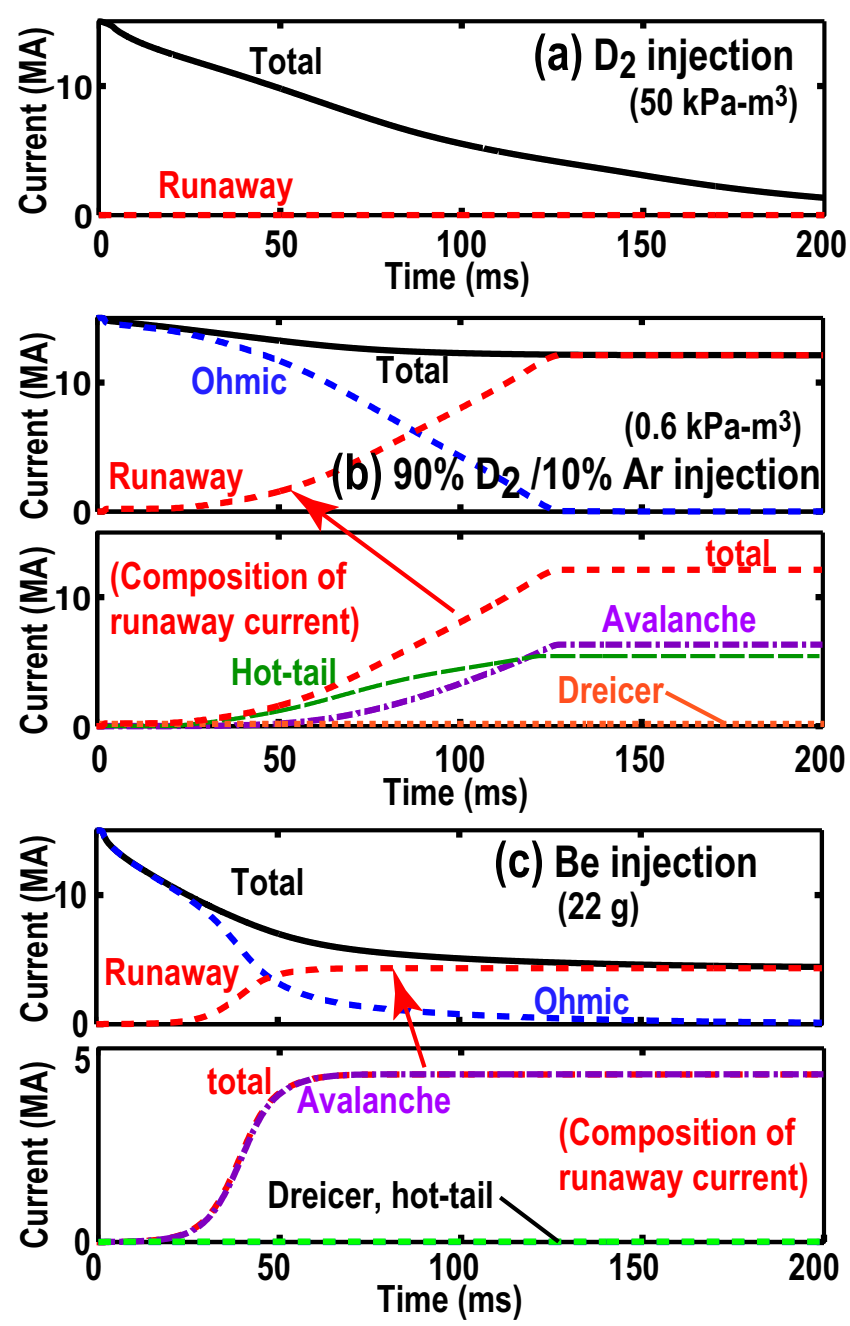

FIG. 7. 1D (GO) simulations of ITER RE current evolution for different rapid shutdown scenarios: (a) massive $\mathrm{D}_{2}$ injection, (b) weaker $\mathrm{D}_{2} / \mathrm{Ar}$ injection, and (c) weak Be injection. completely dominated by avalanche amplification, resulting in a RE plateau current of slightly less than $5 \mathrm{MA}$. In the 1D simulations of Fig. 7, the TQ is produced entirely by the inward diffusion of impurities from the plasma edge. The TQ MHD is not treated and therefore the extended hot tail production of Fig. 7(b) is possible, where an inward moving cold front produces hot tail seeds over an extended time period. Also, the TQ MHD prompt losses of REs are not included. Despite these approximations, models such as this serve to illustrate that RE seed formation can be important for determining the final RE plateau current level in ITER. Reduced RE current formation such as seen in Fig. 7(c) has also been predicted previously in different simulations of $\mathrm{Be}$ and Li pellet shutdown of ITER. ${ }^{60,61}$

Experimentally, there is some difficulty measuring the importance of different RE seed terms in different experiments. Some estimates indicate that Dreicer seeds can explain observed RE levels, while other machines have indicated that hot tail formation or large pre-existing RE levels need to be invoked to explain observed disruption RE levels. A $B=2 \mathrm{~T}$ toroidal field threshold for RE formation was initially observed in JET, JT60, and Tore Supra, but then more recent experiments indicated that this was not an absolute threshold. $^{49,62}$ There is a strong trend across experiments that higher- $Z$ injection shutdown creates more RE seeds, with low $\mathrm{Z}$ MGI $\left(\mathrm{He}, \mathrm{D}_{2}\right)$ rarely creating significant $\mathrm{RE}$ seeds. ${ }^{39,49}$

\section{Runaway electron prompt loss}

The simulations of Fig. 7 ignore loss of REs to the wall; it is not certain to what degree this is accurate for ITER. In present devices, a significant fraction of the RE seed population formed in the TQ appears to be lost to the wall at the end of the TQ. MHD simulations indicate that this prompt loss is due to TQ island overlap and destruction of nested magnetic surfaces. This prediction is consistent with experiments showing larger RE loss levels in elongated discharges (which have more island overlap) as opposed to circular discharges. ${ }^{63}$ Prompt loss also appears larger, on average, in smaller machines. The simulations also support thisindicating smaller prompt loss with increasing machine size. In ITER, the simulations indicate almost no TQ RE prompt loss, suggesting that this RE loss mechanism will be small there. ${ }^{64}$ Examples of post-TQ flux surface structures simulated by NIMROD in C-MOD, DIII-D, and ITER are shown in Fig. 8. It can be seen that there are larger regions of good flux surfaces (less stochastic regions) in ITER than in the smaller machines.

\section{Runaway electron amplification}

According to avalanche theory, runaway electron current is expected to grow in time with a rate $\nu=\nu_{R}-\nu_{D}$, where the growth term $\nu_{R}$ is due to avalanching, while the $\nu_{D}$ term is due to collisional drag on target electrons. During the $\mathrm{CQ}$, the term $\nu_{D}$ is typically small, and the RE current is expected to grow rapidly. Qualitative evidence of the validity of this equation has been achieved during the CQ; Lack of in-situ measurement of the RE seed term size has made 
(a) C-MOD

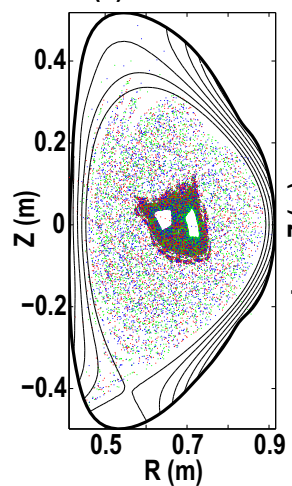

(b) DIII-D

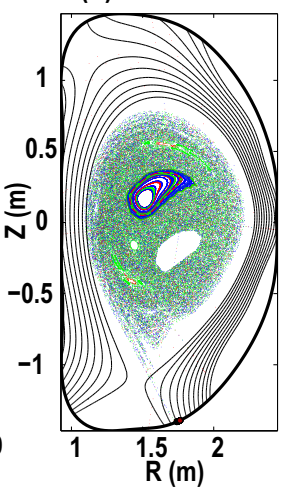

(c) ITER

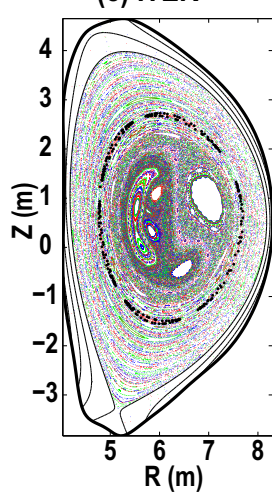

FIG. 8. NIMROD simulations of post-TQ flux surface structure in (a) C-MOD, (b) DIII-D, and (c) ITER. ${ }^{89}$

quantitative confirmation challenging though. ${ }^{10}$ In the RE plateau, where the measured total plasma current $I_{P}$ can be assumed to be dominated by REs, quantitative comparisons between avalanche theory with experiment were made in DIII-D. It was found that there was an anomalous loss term of about $10 \mathrm{~s}^{-1}$ (i.e., the $\nu_{D}$ term is much larger than expected), while the $\nu_{R}$ (growth) term seems to be correct. ${ }^{65}$ It is unclear at present to what extent these results are applicable during the CQ, e.g., to what extent plateau RE current dissipation reflects CQ RE current dissipation.

\section{Runaway electron plateau}

Sufficiently large RE seeds can be amplified to the point where the RE current takes over the plasma current, generating a long-lived RE plateau. Presently, post-disruption ITER RE plateaus of about 10-12 MA are typically assumed, ${ }^{1}$ although simulations (such as Fig. 7) indicate that there will be a large variation in this number depending on the shutdown scenario. The characteristics of the RE plateau are important for attempting to dissipate it in a controlled manner. Soft X-ray (SXR) measurements have shown that the RE plateau tends to form a narrow circular beam $(a<1 \mathrm{~m}$ in DIII$\mathrm{D})$. The RE beam coexists with a cold $\left(T_{e} \approx 1-2 \mathrm{eV}\right)$ background plasma. ${ }^{67}$ Neutrals appear to be mostly excluded from the center of the RE beam.

Measurements of the energy of the plateau REs have been made, indicating a broad energy distribution going from $\mathrm{keV}$ to tens of MeV. In DIII-D, SXR and hard X-ray (HXR) brightness measurements were used to diagnose the core of the RE beam. The number of fast electrons at low energies $(1-10 \mathrm{keV})$ was found to be much higher than expected from avalanche theory, ${ }^{68}$ as shown in Fig. 9(c). ${ }^{67}$ In JET, HXR pulse-height counting was used to estimate the core RE beam energy. Peak RE energies of order $15 \mathrm{MeV}$ were observed. Two distinct energy components were inferred in some cases, possibly due to primary and secondary RE components. ${ }^{69}$ In TEXTOR, scintillating and calorimetric probes have been used to diagnose the edge RE beam energy, finding a distribution function with mean energy of order $5 \mathrm{MeV},{ }^{70,71,88}$ Figs. 9(a) and 9(b). Estimates of the total energy of the RE beam typically give values ranging from $30 \mathrm{~kJ}$ in TEXTOR to several $100 \mathrm{~kJ}$ in $\mathrm{JET},{ }^{54}$ with a linear
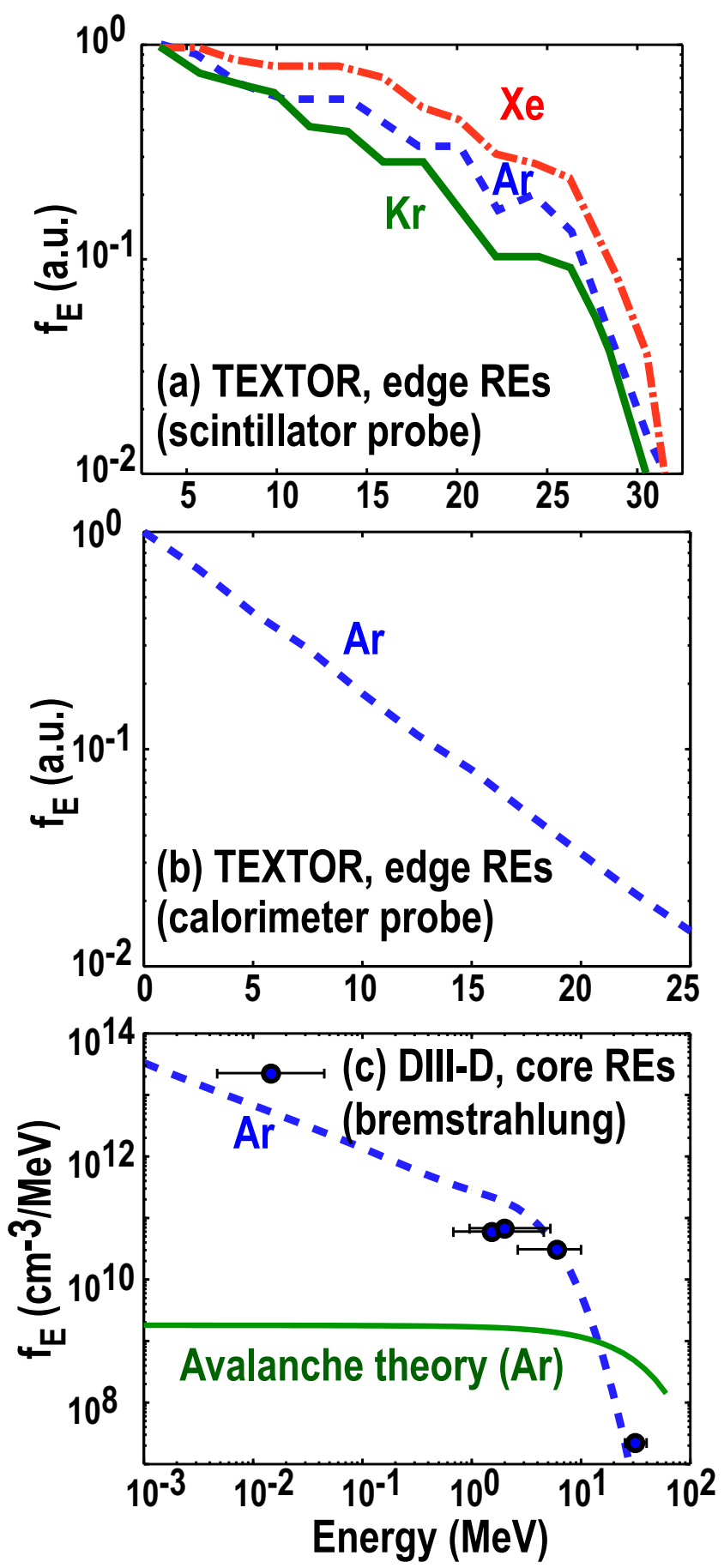

FIG. 9. Plateau or near-plateau RE energy distribution estimates from (a) edge of TEXTOR after $\mathrm{Ar}, \mathrm{Kr}$, or Xe MGI shutdown using scintillator probe ${ }^{70}$ (b) edge of TEXTOR after Ar MGI shutdown using calorimeter probe; and (c) core of DIII-D after Ar pellet shutdown. Reproduced by permission of IAEA, Vienna from E. M. Hollmann et al., Nucl. Fusion 53, 083004 (2013). Copyright 2013 IOP Publishing and M. Forster et al., Nucl. Fusion 51, 043003 (2011). Copyright 2011 IOP Publishing.

increase in RE beam kinetic energy with RE current. $^{72}$ Magnetic energy is typically estimated as being larger than the kinetic energy. Pitch angle estimates of RE beams have been made, giving $\vartheta \approx 0.1-0.2$ radians based on the elongation of synchrotron images in TEXTOR ${ }^{73}$ and DIII-D. ${ }^{74}$ Interpretation of microwave ECE spectra suggested a higher 
pitch angle (close to $\sin (\vartheta) \sim 1)$ for low energy $(\sim 100 \mathrm{keV}$ range) REs in DIII-D.

\section{Runaway electron final loss}

Understanding the dynamics of the final loss of a RE beam striking the vessel wall is important for minimizing the resulting damage. ${ }^{78} \mathrm{RE}$ beams interacting with the wall are typically lost on a timescale $\sim 1 \mathrm{~ms}$ in present tokamaks. ${ }^{67}$ Often, multiple HXR spikes are observed, suggesting a repetitive instability. RE beam/wall strikes have been observed in all directions (up, down, inner wall, and outer wall). Especially worrisome is the observation that RE beams can cause deep localized damage in wall tiles. Post mortem analysis shows RE damage to tiles which is often almost circular, not grazing incidence as one would expect from a smooth scrape-off of the RE beam. Also, RE beam strikes have been observed in locations apparently in shadowed wall regions, ${ }^{66}$ suggesting some form of kink during the final loss phase. In some machines, e.g., DIII-D, loss to the outer midplane often seems more sudden and unstable than loss to the inner midplane; this may be due to error fields (field ripple), outward drift orbit shifts, or changes in loop voltage characteristics for outward versus inward RE motion.

Data on the wetted area during the RE final loss is very sparse. Some IR images have been captured in JET, indicating multiple strike points on tile leading edges, with spot sizes of order $10 \mathrm{~cm}^{75}$ A spatial array of HXR sensors in DIII-D indicated a strong toroidal variation in the HXR emission, consistent with a kink. ${ }^{63}$

$\mathrm{RE}$ damage to the wall has been simulated by various codes. It has been found that deep $(>1 \mathrm{~mm})$ melt damage to Be wall tiles is expected to occur following a strike with a highenergy RE beam. Damage is highly sensitive to RE pitch angle and energy. Higher energies can actually reduce melt layer thickness. ${ }^{76}$ The melt layer is expected to persist for a long time ( $0.2 \mathrm{~s}$ ), so splashing or flowing is a concern. ${ }^{77}$ Evaporation is shown to reduce the melt layer depth significantly (by several times). ${ }^{77}$ Additionally, at high impact angle and high energy, melting of cooling line braze joints could occur. ${ }^{78}$

RE beam energy is thought to be largely magnetic in present experiments. This is expected to be more the case in ITER, since (assuming a fixed RE energy distribution) $W_{\text {kin }} \sim I_{P}$ but $W_{\text {mag }} \sim I_{P}^{2}{ }^{72,79}$ Because of this, the behavior of the magnetic energy during the final loss is extremely important. Experiments indicate that inward moving RE beams will tend to have an increased toroidal electric field, causing increased $W_{k i n}$. Additionally, the loss of current as the RE beam moves into the wall causes a loop voltage rise which may increase the peak RE energy. Experiments indicate that slower motion of RE beams into the wall results in greater conversion of kinetic into magnetic energy, while fast motion $\tau_{\text {Loss }}<\tau_{\text {Wall }}, \tau_{\text {Ohmic }}$ results in RE current dominantly converted into ohmic current and vessel current; this also consistent with $0 \mathrm{D}$ and 1D simulations. ${ }^{79,80}$

\section{B. Active mitigation of runaway electrons}

A wide range of creative methods for mitigating REs have been proposed, ranging from sacrificial limiters to dropped bricks and swinging probes. Because of the very limited machine access of ITER, though, it appears that many of these methods will be impractical, limiting RE mitigation to impurity injection or magnetic coil ramps.

\section{Runaway mitigation with applied non-axisymmetric fields}

Externally applied non-axisymmetric magnetic fields have been shown to be useful in tokamaks for affecting resistive wall modes and suppressing edge localized modes. Intentional external magnetic perturbations have also been applied to all phases of rapid shutdowns to try to affect the dynamics. In JT-60, application of an $n=2$ field was shown to have some effect on final RE plateau current levels. In TEXTOR, an applied $n=3$ field had a clear effect on final RE levels. ${ }^{81}$ In DIII-D, both $n=1$ and $n=3$ were attempted: $n=3$ had no observable effect and $n=1$ may have had a small unreliable effect. ${ }^{82}$ Similar experiments were tried in JET, but no clear effect was seen.

Simulations of the effect of applied non-axisymmetric magnetic fields on REs in ITER have been done, both by current channel (DINA) plus particle tracking (DRIFT) simulations $^{83}$ and by 3D full- $f$ Monte Carlo (ANTS code) simulations. ${ }^{84}$ These simulations are in agreement that significant (up to 100\%) losses can be achieved for REs at large minor radius, but very little effect is achieved on REs near the center of the current channel. Both simulations indicated that the loss pattern of the REs lost to the wall could be affected by the applied field errors. The DINA simulations indicated that the structure of the external wall (ports, test blanket modules, etc.) and the motion of the plasma current channel (VDE) were the dominant factors in the RE loss pattern, rather than the applied field errors.

\section{Runaway plateau position control}

After forming, RE plateaus tend to drift inward slightly and then get lost vertically (with the exception of Tore Supra, where loss tends to be radial). The initial inward motion is thought to be due to the drop in plasma current during the CQ (coupled with the vertical field in the vessel); while the subsequent vertical motion is thought to be due to the decaying RE plasma current creating image currents in the vessel wall (resulting in a linear vertical motion, rather than the exponential motion from standard vertical loss due to plasma elongation). This RE plateau motion can be, in principle, opposed by sufficiently strong control coils. Vertical control has been demonstrated in Tore-Supra and DIII-D, ${ }^{90}$ and radial control has been demonstrated in Tore Supra. ${ }^{91}$

Because of the vertical asymmetry in the vacuum vessel and also in the plasma shape, the CQ plasma, and RE plateau will tend to move vertically up in ITER. Theoretically, the situation is improved if the plasma can be pre-emptively moved down to the true neutral point for CQ vertical motion: in this case, the resulting vertical displacement can be kept small (depending on the scatter in post-TQ vertical position seen in all disruptions). Consistent with this, DINA simulations of ITER disruptions found that better RE plateau 
control could be achieved if there was sufficient precursor time $(>1 \mathrm{~s})$ to move the plasma down by $12 \mathrm{~cm}$. In this case, RE plateau vertical control could be achieved for $I_{P}>9.7$ MA (as compared with $I_{P}>14$ MA without the $12 \mathrm{~cm}$ move). Figure 10(a) shows an example of a vertically uncontrolled case, while Fig. 10(b) shows an example of a vertically controlled case. For centered, slowly decaying RE beams, the simulations indicate that vertical control can be maintained for a drop in $I_{P}$ down to 2 MA. ${ }^{85}$

In addition to the vertical loss of the RE plateau, it is assumed that decay of the RE current (due to unknown levels of collisional damping or diffusive loss of REs in ITER) will tend to move the RE current channel inward toward the center post, as is observed in present experiments. Predisruption inward motion of the ITER plasma may be desirable, as this could limit the plasma on the inboard midplane pre-emptively, dropping elongation and reducing post-TQ vertical motion. Inward motion of the RE plateau is probably not desirable, possibly leading to repeated beryllium tile melting on the midplane center post. The ability to retain radial control of the RE plateau in ITER will depend on the decay rate of RE current. For example, for $I_{P}=10 \mathrm{MA}$, the minimum radially controllable loss time for $\mathrm{RE}$ current is $340 \mathrm{~ms}$, according to DINA simulations.

\section{Runaway electron collisional suppression}

Sufficiently large amounts of material can drag on REs to the point where avalanche amplification can be suppressed in
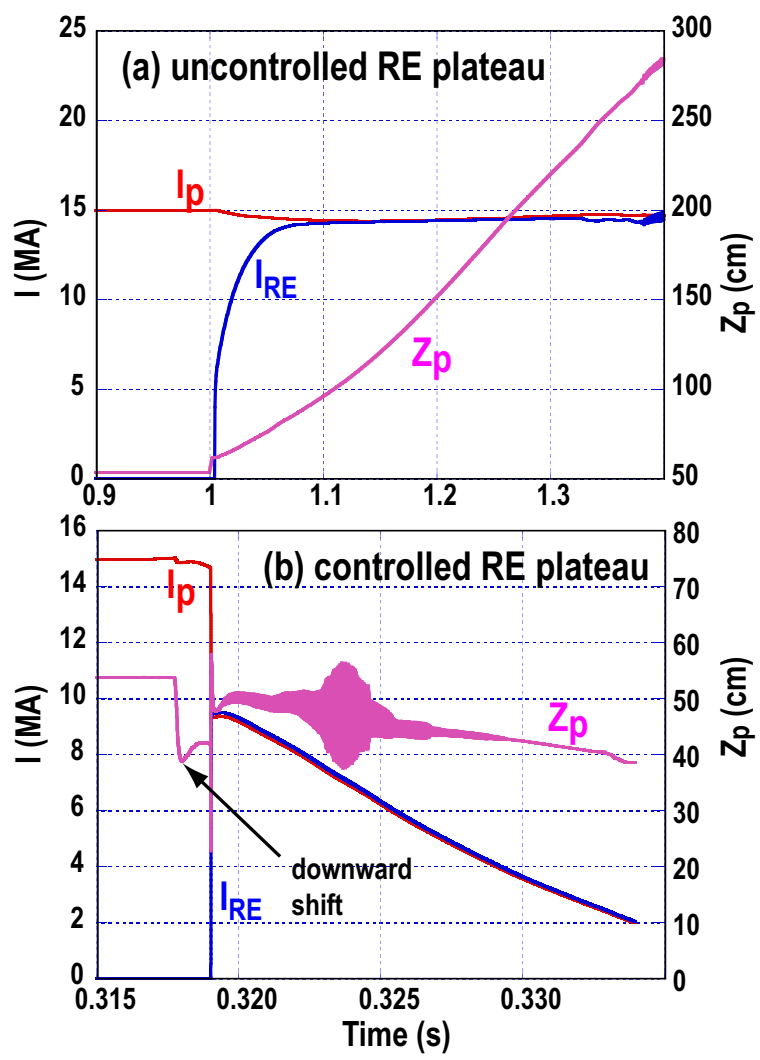

FIG. 10. Time traces of DINA simulations of ITER RE plateau control showing (a) vertically lost RE plateau and (b) shot with pre-emptive downward shift and subsequent controlled RE plateau (assuming RE loss time of $400 \mathrm{~ms})$. the CQ. The predicted densities required are very high, with total electron densities of order $n_{\text {crit }}=10^{22} / \mathrm{m}^{3}$ required during the middle of the $\mathrm{CQ}$, when high toroidal electric fields (up to $E_{\varphi} \approx 100 \mathrm{~V} / \mathrm{m}$ ) exist. Present experiments using MGI or SPI have reached up to $20 \%$ of the mid-CQ $n_{\text {crit }}=$ $10^{22} / \mathrm{m}^{3}$ Reaching $100 \%$ of mid-CQ $n_{\text {crit }}$ appears challenging, probably requiring very specialized highly refined mass deposition methods like finely tuned SPI or LSP, but is possible, in principle, if "ideal" high-Z deposition can be realized, as shown by the squares in Fig. 11(a). The simulations ignore CQ particle transport; there is an indication from SPI experiments that there is a CQ transport loss of impurities which is not negligible, and this would make it even more difficult to reach $100 \%$ mid-CQ $n_{\text {crit }}$. Additionally, the "ideal" impurity deposition is predicted to result in unacceptably short CQ durations, as shown in Fig. 11(b).

Although mid-CQ collisional avalanche suppression has not been achieved in present tokamaks, a counteracting of the RE avalanche does appear to have been achieved during the RE plateau, where toroidal electric fields are significantly $(>10 \times)$ weaker. Examples are shown in Fig. 12 for (a) DIII$\mathrm{D}$ and (b) Tore-Supra, where large quantities of gas are injected into the RE plateau, resulting in decay of the RE current. In both cases, high- $Z$ gas is more effective at reducing RE current. The details of the mechanisms for this enhanced dissipation of RE current are not clear at the moment (e.g., if electron-electron collisional drag is responsible, electron-ion drag, or some multi-step process such as collisions leading to increased radial transport).

\section{4. $C Q M H D$ destabilization}

It is well known that RE confinement can be strongly degraded by MHD instabilities. It has been proposed that

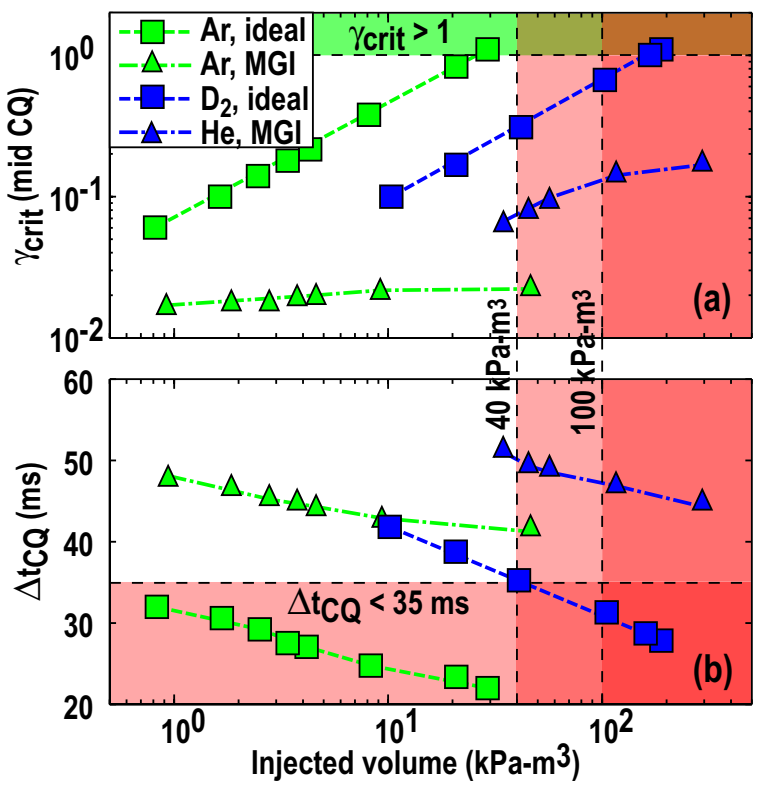

FIG. 11. OD (KPRAD) simulations of rapid shutdown characteristics in ITER as a function of injected gas volume showing (a) mid-CQ collisional RE suppression fraction $\gamma_{\text {crit }} \equiv n_{\text {tot }} / n_{\text {crit }}$ and (b) CQ duration. Ideal deposition means gas is deposited directly into core in $1 \mathrm{~ms}$ pulse; MGI deposition means gas is delivered using $5 \mathrm{~ms}$ tube and is assumed to diffuse into core from edge with diffusion coefficient $D_{\perp}=1 \mathrm{~m}^{2} / \mathrm{s}$. 
sudden impurity injection during the CQ could de-stabilize MHD due to current profile shrinking, thus enhancing RE loss to the wall sufficiently to counteract the CQ avalanche gain. ${ }^{86}$ First, experiments attempting to demonstrate this have been performed at Tore-Supra. Burst disk MGI was used to fire $100 \mathrm{~Pa}-\mathrm{m}^{3}$ of neon into the CQ of plasmas shut down by slow Ar or $\mathrm{D}_{2}$ puffing. Some MHD events (current flattening) and RE loss events were seen during the $\mathrm{CQ}$, but no clear correlation with the presence or absence of the midCQ neon injection was observed. ${ }^{21}$ As a possible explanation of this observation, it was suggested that perturbation of the CQ current channel is extremely difficult via MGI, even in the absence of RE current, due to Townsend breakdown of the neutral gas. ${ }^{87}$ Additionally, the already very low CQ electron temperature could make it hard to create a large perturbation to the plasma conductivity via gas injection.

\section{DISCUSSION}

Design of the DMS for ITER is a complex problem with many performance requirements and engineering constraints which are to some degree contradictory. For example, injecting some high-Z material in the pre-TQ phase seems desirable to reduce TQ conducted heat loads, but this then shortens the CQ time undesirably and increases RE seed formation.
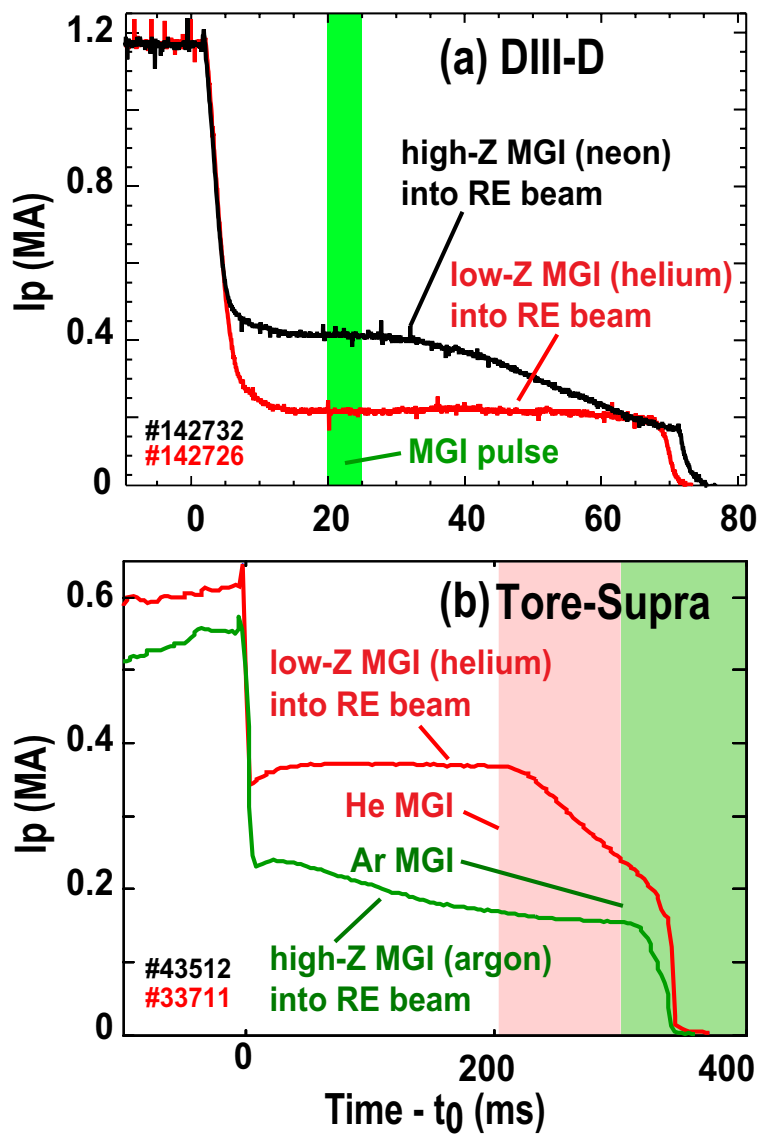

FIG. 12. Dissipation of RE current with MGI into RE plateau demonstrated in (a) DIII-D and (b) Tore Supra. ${ }^{91}$ Reproduced by permission of IAEA, Vienna from E. M. Hollmann et al., Nucl. Fusion 53, 083004 (2013). Copyright 2013 IOP Publishing.
DMS shutdown therefore needs to be designed with the entire disruption in mind, with priorities placed on which forms of wall damage are more tolerable than others. A possible prioritization could be: (1) keep CQ times longer than $35 \mathrm{~ms}$ (to avoid mechanical damage to shielding blanket modules); (2) avoid high energy RE-wall strikes (since a cooling line leak into the vacuum chamber, while not a safety event, could be very challenging to repair and result in an extended repair period); and (3) minimize first wall heat loads. Heat loads and surface melting could result in reduced wall lifetime and a shortened run period, but would most likely not result in catastrophic machine closure, and could therefore be assigned a lower priority.

Given this prioritization, it seems that a good approach for the ITER DMS system would be: (1) low-Z (with possible trace high-Z) injection during the pre-TQ to somewhat reduce TQ conducted heat loads but keep a long CQ; followed by (2) high-Z injection during the CQ and/or RE plateau to attempt to minimize RE current and reduce peak RE energy. The overall timing and quantities of these mass injections which best accomplish the mitigation goals of ITER in different scenarios (e.g., rotating plasma vs nonrotating locked mode target plasma) is unknown. Additionally, the optimum injection valve geometry is still uncertain. Limited present modeling and experiments tend to suggest an improved TQ radiation heat load uniformity when using more toroidally spaced injection ports; with the exception of C-MOD, which has observed suggestions of increased heat load localization with multiple toroidal valves, possibly due to increased TQ MHD levels. The role of TQ MHD and plasma rotation and flows on the TQ heat loads are still poorly studied and merit continued research. Additionally, only preliminary simulations and experiments are available on the effects of poloidally spaced injection valves on TQ heat load uniformity; although first results from AUG and DIII-D in this area are encouraging.

For RE mitigation, present data clearly demonstrate the effectiveness of injected impurities in reducing RE current when injected during the plateau, but no clear effect has been demonstrated when injecting during the CQ. Increasing RE plateau current dissipation could be beneficial or detrimental: simulations show that the increasing the RE current decay rate will increase the vertical instability rate, which will in turn (undesirably) increase the RE current at the REwall impact time, but will also (desirably) decrease the fraction of RE plateau magnetic energy which is converted into kinetic energy. The optimum quantity of and timing of injected impurities for minimizing wall damage may therefore not be readily apparent without modeling the RE beamwall impact process.

Various types of injection hardware have been studied, but the optimum impurity injection method or methods for the ITER DMS system remain to be decided. For TQ mitigation, rapid "trigger-to-TQ" times of $20 \mathrm{~ms}$ or less are desirable, which would seem to mandate installation of the injection hardware close to the plasma. However, experience with present fast valves indicates that it is sometimes desirable to perform maintenance on them (due to valve seat damage, for example). It may therefore be prudent to install the 
injector hardware farther (4 or $7 \mathrm{~m})$ from the vessel. Further research on rapid, reliable pellet acceleration may be advantageous in this regard: for example, SPI using a $1000 \mathrm{~m} / \mathrm{s}$ pellet (as opposed to the present $200 \mathrm{~m} / \mathrm{s}$ ) could give a TQ onset time of order $5 \mathrm{~ms}$ even if fired from a port cell.

The relative benefits of different impurity injection methods (MGI vs SPI) on TQ radiation structure have only begun to be investigated, and making an informed decision on which technique gives best radiation symmetry will be challenging. For example, rapid injection (SPI or LSP) gives better core deposition and (apparently) lower MHD, but also less time for impurities to spread toroidally prior to the TQ when compared with MGI. The benefits of using dust (such as could be delivered with LSP) for TQ and RE mitigation have not been investigated at all, either experimentally or theoretically, despite some possible anticipated benefits such as reduced pumping system loads and improved CQ impurity assimilation.

In addition to the sequence and method of injecting impurities delivered by the DMS, the optimum sequence of coil inputs to be programmed for disruption mitigation will need to be determined for ITER. Plasma position control in ITER will probably be too slow to have much effect on the $\mathrm{TQ}$, and simulations indicate that even position control of the RE plateau will be difficult in most scenarios. The usefulness of non-axisymmetric coil inputs is also uncertain at present: to-date only some machines show clear effect on RE loss from using toroidally asymmetric field errors, and simulations presently suggest that only REs in the very edge of the ITER current channel can be affected by intentional field errors. In the TQ, simulations indicate that intentional field errors could enhance toroidally localized heat loads, and there are some preliminary indications of this effect in DIII$\mathrm{D}$ experiments, so this may be an undesirable side effect of intentional static field errors. Overall, the benefit of intentional static field errors on disruption mitigation in ITER is not clear at present but certainly warrants further study.

In summary, ITER will require a working DMS from the beginning of its operation. Some low power/low current shots can be expected initially for testing of the DMS, but since even $5 \mathrm{MA}$ of unmitigated RE current could cause wall damage, ITER will early on enter a regime where a working DMS will be essential. Research in support of the design of the ITER DMS is ongoing and significant progress has been made in recent years; an attempt has been made to summarize some of this research here. Given the complexity of this problem, its importance to ITER, and the large number of remaining uncertainties in extrapolating present results to ITER, it seems unlikely that the best possible DMS design for ITER will be determined by 2017. It will probably therefore be prudent to design the ITER DMS with as much flexibility as allowable, to best be able to take advantage of future experimental and modeling advances in this area.

\section{ACKNOWLEDGMENTS}

Assistance of Dr. N. Eidietis explaining ITER coil capabilities as well as useful comments on this manuscript by Dr. D. Campbell and Dr. P. Parks are gratefully acknowledged. This work was supported in part by the U.S. Department of Energy under DE-FG02-07ER54917 and in part by the Max-Planck/Princeton Center for Plasma Physics. The views and opinions expressed herein do not necessarily reflect those of the ITER Organization.

${ }^{1}$ T. C. Hender, J. C. Wesley, J. Bialek, A. Bondeson, A. H. Boozer, R. J. Buttery, A. Garofalo, T. P. Goodman, R. S. Granetz, Y. Gribov, O. Gruber, M. Gryaznevich, G. Giruzzi, S. Guenter, N. Hayashi, P. Helander, C. C. Hegna, D. F. Howell, D. A. Humphreys, G. T. A. Huysmans., A. W. Hyatt, A. Isayama, S. C. Jardin, Y. Kawano, A. Kellman, C. Kessel, H. R. Koslowski, R. J. La Haye, E. Lazzarus, Y. Q. Liu, V. Lukash, J. Manickam, S. Medvedev, V. Mertens, S. V. Mirnov, Y. Nakamura, G. Navratil, M. Okabayashi, T. Ozeki, R. Paccagnella, G. Pautasso, F. Porcelli, V. D. Pustovitov, V. Riccardo, M. Sato, O. Sauter, M. J. Schaffer, M. Shimada, P. Sonato, E. J. Strait, M. Sugihara, M. Takechi, A. D. Turnbull, E. Westerhof, D. G. Whyte, R. Yoshino, H. Zohm, and ITPA MHD Topical Group, Nucl. Fusion 47, S128 (2007).

${ }^{2}$ B. Cannas, A. Fanni, G. Pautasso, G. Sias, and ADEX Upgrade Team, Fusion Eng. Des. 86, 1039 (2011).

${ }^{3}$ E. M. Hollmann, G. Arnoux, N. Commaux, N. W. Eidietis, T. E. Evans, R. S. Granetz, A. Huber, D. A. Humphreys, V. A. Izzo, A. N. James, T. C. Jernigan, M. Lehnen, G. Maddaluno, R. Paccagnella, P. B. Parks, V. Philipps, M. L. Reinke, D. L. Rudakov, F. Saint-Laurent, V. Sizyuk, E. J. Strait, J. C. Wesley, C. P. C. Wong, and J. H. Yu, J. Nucl. Mater. 415, S27 (2011).

${ }^{4} \mathrm{M}$. Sugihara, "Status of DMS and other disruption related issues in ITER," paper presented at 21st ITPA MHD Workshop, San Diego, USA, 2012

${ }^{5}$ S. Maruyama, S. Putvinski, M. Sugihara, G. Kiss, R. Marrs, B. Macdonald, and P. Edwards, "ITER disruption mitigation requirements and development of a gas cartridge concept," in IEEE/NPSS 24th Symposium on Fusion Engineering (2011). See http://ieeexplore.ieee.org/ stamp/stamp.jsp?tp $=$ \&arnumber $=6052232$.

${ }^{6}$ J. Roth, E. Tsitrone, A. Loarte, Th. Loarer, G. Counsell, R. Neu, V. Philipps, S. Berzinsek, M. Lehnen, P. Coad, Ch. Grisolia, K. Schmid, K. Krieger, A. Kallenbach, B. Lipschultz, R. Doerner, R. Causey, V. Alimov, W. Shu, O. Ogorodnikova, A. Kirschner, G. Federici, A. Kukushkin, and EFDA PWI Task Force, J. Nucl. Mater. 390, 1 (2009).

${ }^{7}$ P. L. Taylor, A. G. Kellman, T. E. Evans, D. S. Gray, D. A. Humphreys, A. W. Hyatt, T. C. Jernigan, R. L. Lee, J. A. Leuer, S. C. Luckhardt, P. B. Parks, M. J. Schaffer, D. G. Whyte, and J. Zhang, Phys. Plasmas 6, 1872 (1999).

${ }^{8}$ G. Pautasso, K. Buchl, J. C. Fuchs, O. Gruber, A. Herrmann, K. Lackner, P. T. Lang, K. F. Mast, M. Ulrich, and H. Zohm, Nucl. Fusion 36, 1291 (1996).

${ }^{9}$ N. Commaux, L. R. Baylor, T. C. Jernigan, E. M. Hollmann, P. B. Parks, D. A. Humphreys, J. C. Wesley, and J. H. Yu, Nucl. Fusion 50, 112001 (2010).

${ }^{10}$ E. M. Hollmann, N. Commaux, N. W. Eidietis, T. E. Evans, D. A Humphreys, A. N. James, T. C. Jernigan, P. B. Parks, E. J. Strait, J. C. Wesley, J. H. Yu, M. E. Austin, L. R. Baylor, N. H. Brooks, V. A. Izzo, G. L. Jackson, M. A. van Zeeland, and W. Wu, Phys. Plasmas 17, 056117 (2010)

${ }^{11}$ Z. Yong-Zhen, F. Xing-Ya, G. Gan-Cheng, X. De-Ming, and Z. Yin-Jia, Chin. Phys. 15, 2053 (2006)

${ }^{12}$ P. B. Parks, M. N. Rosenbluth, S. V. Putvinski, and T. E. Evans, Fusion Technol. 35, 267 (1999).

${ }^{13}$ I. N. Bogatu, J. R. Thompson, S. A. Galkin, J. S. Kim, and HyperV Technologies Team, "Disruption mitigation with plasma jets for ITER," in IAEA Conference (2010), poster No. EXS/P2-01.

${ }^{14}$ E. M. Hollmann, T. C. Jernigan, P. B. Parks, J. A. Boedo, T. E. Evans, M. Groth, D. A. Humphreys, A. N. James, M. J. Lanctot, D. Nishijima, D. L. Rudakov, H. A. Scott, E. J. Strait, M. A. van Zeeland, J. C. Wesley, W. P. West, W. Wu, and J. H. Yu, Nucl. Fusion 48, 115007 (2008).

${ }^{15}$ K. H. Finken, M. Lehnen, and S. A. Bozhenov, Nucl. Fusion 51, 033007 (2011).

${ }^{16}$ P. B. Parks and W. Wu, Nucl. Fusion 51, 073014 (2011).

${ }^{17}$ J. C. Jernigan, L. R. Baylor, S. K. Combs, C. R. Foust, E. M. Hollmann, D. A. Humphreys, P. B. Parks, and J. C. Wesley, "New valve for massive gas injection in DIII-D," Bull. Am. Phys. Soc. 51, 271 (2006).

${ }^{18}$ L. R. Baylor, S. K. Combs, C. R. Foust, T. C. Jernigan, S. J. Meitner, P. B Parks, J. B. Caughman, D. T. Fehling, S. Maruyama, A. L. Qualls, D. A. Rasmussen, and C. E. Thomas, Nucl. Fusion 49, 085013 (2009). 
${ }^{19}$ G. Pautasso, M. Bernert, K. Gal, K. Mank, A. Mlynek, and A. Herrmann, "Toward the density required for runaway electron suppression in ITER," in Proceedings of the 24th IAEA Fusion Energy Conference (2012), poster No. EX/P8-12.

${ }^{20}$ S. K. Combs, S. J. Meitner, L. R. Baylor, J. Caughman, N. Commaux, D. T. Felding, C. R. Foust, T. C. Jernigan, J. M. McGill, P. B. Parks, and D. A. Rasmussen, "Alternative techniques for injecting massive quantities of gas for plasma-disruption mitigation," IEEE Trans. Plasma Sci. 38, 400 (2010).

${ }^{21}$ F. Saint-Laurent, G. Martin, T. Alarcon, A. Le Luyer, P. B. Parks, P. Pastor, S. Putvinski, J. Bucalossi, S. Bremond, and Ph. Moreau, Fusion Sci. Technol. 64, 711 (2013).

${ }^{22}$ M. J. Gouge, S. K. Combs, P. W. Fisher, and S. L. Milora, Rev. Sci. Instrum. 60, 570 (1989).

${ }^{23}$ S. K. Combs, L. R. Baylor, S. J. Meitner, N. Commaux, T. C. Jernigan, D. A. Rasmussen, S. L. Milora, and T. R. Younkin, "R\&D in support of the shattered pellet technique for disruption mitigation," in VLT Conference All Presentation (Oak Ridge National Laboratory, 2013). See http:// vlt.ornl.gov/research/20130619_VLT_combs.pdf.

${ }^{24}$ R. A. Pitts, S. Carpentier, F. Escourbiac, T. Hirai, V. Komarov, A. S. Kukushikin, M. Merola, R. Mitteau, and P. C. Stangeby, "A full tungsten divertor for ITER," J. Nucl. Mater. 438, S48 (2013).

${ }^{25}$ A. Loarte, G. Saibene, R. Sartori, V. Riccardo, P. Andrew, J. Paley, W. Fundamenski, T. Eich, A. Herrmann, G. Pautasso, A. Kirk, G. Counsell, G. Federici, G. Strohmayer, D. Whyte, A. Leonard, R. A. Pitts, I. Landman, B. Bazylev, and S. Pestchanyi, Phys. Scr. T128, 222 (2007).

${ }^{26}$ P. C. de Vries, G. Arnoux, A. Huber, J. Flanagan, M. Lehnen, V. Riccardo, C. Reux, S. Jachmich, C. Lowry, G. Calabro, D. Frigione, M. Tsalas, N. Hartmann, S. Brezinsek, M. Clever, D. Douai, M. Groth, T. C. Hender, E. Hodille, E. Joffrin, U. Kruezi, G. F. Matthews, J. Morris, R. Neu, V. Philipps, G. Sergienko, M. Sertoli, and JET EFDA Contributors, Plasma Phys. Controlled Fusion 54, 124032 (2012).

${ }^{27}$ M. Lehnen, G. Arnoux, N. Hartman, S. Brezinsek, S. Devaus, A. Huber, S. Jachmich, U. Kruezi, G. F. Matthews, C. Reux, V. Riccardo, B. Sieglin, M. F. Stamp, and P. C. de Vries, J. Nucl. Mater. 438, S102 (2013).

${ }^{28}$ V. Philipps, M. Freisinger, A. Huber, and T. Loarer, J. Nucl. Mater. 390, 478 (2009).

${ }^{29}$ B. Pegourie, H. Wang, C. Brosset, J. Bucalossi, Y. Corre, T. Dittmar, D. Douai, A. Ekedahl, T. Loarer, C. Reux, F. Saint-Laurent, E. Tsitrone, and S. Vartanian, J. Nucl. Mater. 415, S809 (2011).

${ }^{30}$ E. M. Hollmann, N. A. Pablant, D. L. Rudakov, J. A. Boedo, N. H. Brooks, T. C. Jernigan, and A. Yu. Pigarov, J. Nucl. Mater. 390, 597 (2009).

${ }^{31}$ F. Saint-Laurent, "Control of runaway electron heat loads on Tore Supra," in 38th EPS Conference on Plasma Physics (2011), p. O3.118.

${ }^{32}$ R. S. Granetz, E. M. Hollmann, D. G. Whyte, V. A. Izzo, G. Y. Antar, A. Bader, M. Bakhtiari, T. Biewer, J. A. Boedo, T. E. Evans, I. H. Hutchinson, T. C. Jernigan, D. S. Gray, M. Groth, D. A. Humphreys, C. J. Lasnier, R. A. Moyer, P. B. Parks, M. L. Reinke, D. L. Rudakov, E. J. Strait, J. L. Terry, J. Wesley, W. P. West, G. Wurden, and J. Yu, Nucl. Fusion 47, 1086 (2007).

${ }^{33}$ P. B. Parks and M. N. Rosenbluth, Phys. Plasmas 5, 1380 (1998).

${ }^{34}$ V. Yu. Sergeev, O. A. Bakhareva, B. V. Kuteev, and M. Tendler, Plasma Phys. Rep. 32, 363 (2006).

${ }^{35}$ S. A. Bozhenkov, M. Lehnen, K. H. Finken, G. Bertschinger, H. R. Koslowski, D. Reiter, R. C. Wolf, and TEXTOR Team, Nucl. Fusion 51, 083033 (2011).

${ }^{36} \mathrm{~B}$. Reiter, "Radiative response on massive noble gas injection for runaway suppression in disruptive plasmas," Ph.D. dissertation (University of Munich, 2010).

${ }^{37}$ A. Huber, G. Arnoux, M. N. A. Beurskens, S. A. Bozhenkov, S. Brezinsek, T. Eich, C. Fuchs, W. Fundamenski, S. Jachmich, U. Kreuzi, M. Lehnen, A. Loarte, G. F. Matthews, Ph. Mertens, P. D. Morgan, V. Phikipps, R. A. Pitts, V. Riccardo, U. Samm, B. Schweer, G. Sergienko, M. Stamp, and JET EFDA Contributors, J. Nucl. Mater. 415, S821 (2011).

${ }^{38}$ E. M. Hollmann, N. Commaux, N. W. Eidietis, D. A. Humphreys, T. C. Jernigan, C. J. Lasnier, R. A. Moyer, R. A. Pitts, M. Sugihara, E. J. Strait, J. Watkins, and J. C. Wesley, Phys. Plasmas 20, 062501 (2013).

${ }^{39}$ C. Reux, J. Bucalossi, F. Saint-Laurent, C. Gil, P. Moreau, and P. Maget, Nucl. Fusion 50, 095006 (2010).

${ }^{40}$ E. M. Hollmann, T. C. Jernigan, E. J. Strait, G. Antar, T. E. Evans, D. S. Gray, M. Groth, D. A. Humphreys, P. B. Parks, and D. G. Whyte, Phys. Plasmas 14, 012502 (2007).

${ }^{41}$ A. J. Thornton, K. J. Gibson, I. T. Chapman, J. R. Harrison, A. Kirk, S. W. Lisgo, M. Lehnen, R. Martin, R. Scannell, A. Cullen, and MAST Team, Nucl. Fusion 52, 063018 (2012).
${ }^{42}$ G. M. Olynyk, R. S. Granetz, M. L. Reinke, D. G. Whyte, T. Golfinopolous, J. W. Hughes, J. R. Walk, V. A. Izzo, S. K. Combs, S. L. Milora, and M. W. Brookman, Nucl. Fusion 53, 092001 (2013).

${ }^{43}$ J. Bucalossi, C. Reux, F. Saint-Laurent, M. Becoulet, Y. Corre, P. Devynck, J. L. Gardarein, C. Gil, J. Gunn, G. Huysmans, P. MonierGarbet, J. L. Segui, and E. Tsitrone, J. Nucl. Mater. 415, S832 (2011).

${ }^{44}$ A. J. Thornton, K. J. Gibson, J. R. Harrison, M. Lehnen, R. Martin, A. Kirk, and MAST Team, Plasma Phys. Controlled Fusion 54, 125007 (2012).

${ }^{45}$ V. E. Lukash, A. B. Mineev, and D. Kh. Morozov, Nucl. Fusion 47, 1476 (2007).

${ }^{46}$ V. A. Izzo, Phys. Plasmas 20, 056107 (2013).

${ }^{47}$ E. M. Hollmann, D. A. Humphreys, and P. B. Parks, Nucl. Fusion 52, 033001 (2012).

${ }^{48}$ M. Bakhtiari, Y. Kawano, H. Tamai, Y. Niura, R. Yoshino, and Y. Nishida, Nucl. Fusion 42, 1197 (2002).

${ }^{49}$ M. Lehnen, A. Alonso, G. Arnoux, N. Baumgarten, S. A. Bozhenkov, S. Brezinsek, M. Brix, T. Eich, S. N. Gerasimov, A. Huber, S. Jachmich, U. Kreuzi, P. D. Morgan, V. V. Plyusnin, C. Reux, V. Riccardo, G. Sergienko, M. F. Stamp, and JET EFDA Contributors, Nucl. Fusion 51, 123010 (2011).

${ }^{50}$ G. Pautasso, L. Giannone, O. Gruber, A. Herrmann, M. Maraschek, K. H. Schuhbeck, and ASDEX Upgrade Team, Nucl. Fusion 51, 043010 (2011).

${ }^{51}$ T. E. Evans, A. G. Kellman, D. A. Humphreys, M. J. Schaffer, P. L. Taylor, D. G. Whyte, T. C. Jernigan, A. W. Hyatt, and R. L. Lee, J. Nucl. Mater. 241, 606 (1997).

${ }^{52}$ E. M. Hollmann, D. S. Gray, D. G. Whyte, A. Yu. Pigarov, S. I. Krasheninnikov, J. A. Boedo, and D. A. Humphreys, Phys. Plasmas 10, 2863 (2003).

${ }^{53}$ G. Pautasso, Y. Zhang, B. Reiter, L. Giannone, O. Gruber, A. Herrmann, O. Kardaun, K. K. Khayrutdinov, V. E. Kukash, M. Maraschek, A. Mlynek, Y. Nakamura, W. Schneider, G. Sias, M. Sugihara, and ASDEX Upgrade Team, Nucl. Fusion 51, 103009 (2011).

${ }^{54}$ V. Riccardo, G. Arnoux, P. Cahyna, T. C. Hender, A. Huber, S. Jachmich, V. Kiptily, R. Koslowski, L. Krlin, M. Lehnen, A. Loarte, E. Nardon, R. Paprok, D. Tskhakaya, and JET EFDA Contributors, Plasma Phys. Controlled Fusion 52, 124018 (2010).

${ }^{55}$ S. P. Gerhardt, Nucl. Fusion 53, 023005 (2013).

${ }^{56}$ H. Strauss, R. Paccagnella, J. Breslau, L. Sugiyama, S. Jardin, and R. Sayer, "Sideways wall force produced during disruptions," in IAEA Fusion Energy Conference (2012), poster No. TH/P3-01.

${ }^{57}$ M. Bakhtiari, G. Olynyk, R. Granetz, D. G. Whyte, M. L. Reinke, K. Zhurovich, and V. Izzo, Nucl. Fusion 51, 063007 (2011).

${ }^{58}$ S. Konovalov, P. Aleynikov, Yu. Gribov, A. Ivanov, A. Kavin, R. Khayrutdinov, V. Leonov, V. Lukash, A. Loarte, S. Medvedev, E. Polunovskiy, S. Putvinski, M. Sugihara, and V. Zhogolev, "Characterization of runaway electrons in ITER," in IAEA FEC, ITR/P1-32 (2010).

${ }^{59}$ T. Feher, H. M. Smith, T. Fulop, and K. Gal, Plasma Phys. Controlled Fusion 53, 035014 (2011); G. Papp, T. Fulop, T. Feher, P. C. de Vries, V. Riccardo, C. Reux, M. Lehnen, V. Kiptily, V. V. Plyusnin, B. Alper, and JET EFDA Contributors, Nucl. Fusion 53, 123017 (2013).

${ }^{60}$ V. E. Lukash, R. R. Khayrutdinov, Yu. A. Kareev, and S. V. Mirnov, "Modeling of major disruption modification by fast injection of massive Li pellets in ITER-like tokamak reactor," in IAEA Fusion Energy Conference (2010), poster no. THD/P2-01.

${ }^{61}$ S. V. Konovalov, V. M. Leonov, R. R. Khayrutdinov, V. E. Lukash, S. Yu. Medvedev, S. V. Putvinski, V. E. Zhogolev, P. B. Aleynikov, and A. Kavin, "Studying the capabilities of Be pellet injection to mitigate ITER disruptions," in IAEA Fusion Energy Conference (2012), poster No. ITER/P1-38.

${ }^{62}$ Z. Y. Chen, W. C. Kim, A. C. England, Y. K. Oh, J. G. Kwak, and M. Kwon, "Generation and termination of runaway currents in KSTAR," in 38th EPS Conference on Plasma Physics (2011), p. P1.107.

${ }^{63}$ A. N. James, M. E. Austin, N. Commaux, N. W. Eidietis, T. E. Evans, E. M. Hollmann, D. A. Humphreys, A. W. Hyatt, V. A. Izzo, T. C. Jernigan, R. J. La Haye, P. B. Parks, E. J. Strait, G. R. Tynan, J. C. Wesley, and J. H. Yu, Nucl. Fusion 52, 013007 (2012).

${ }^{64}$ V. A. Izzo, E. M. Hollmann, A. N. James, J. H. Yu, D. A. Humphreys, L. L. Lao, P. B. Parks, P. E. Sieck, J. C. Wesley, R. S. Granetz, G. M. Olynyk, and D. G. Whyte, Nucl. Fusion 51, 063032 (2011).

${ }^{65}$ E. M. Hollmann, P. B. Parks, D. A. Humphreys, N. H. Brooks, N. Commaux, N. Eidietis, T. E. Evans, R. Isler, A. N. James, T. C. Jernigan, J. Munoz, E. J. Strait, C. Tsui, J. Wesley, and J. H. Yu, Nucl. Fusion 51, 103026 (2011). 
${ }^{66}$ R. Nygren, T. Lutz, D. Walsh, G. Martin, M. Chatelier, T. Loarer, and D. Guilhem, J. Nucl. Mater. 241, 522 (1997).

${ }^{67}$ E. M. Hollmann, M. E. Austin, J. A. Boedo, N. H. Brooks, N. Commaux, N. W. Eidietis, D. A. Humphreys, V. A. Izzo, A. N. James, T. C. Jernigan, A. Loarte, J. Martin-Solis, R. A. Moyer, J. M. Munoz-Burgos, P. B. Parks, D. L. Rudakov, E. J. Strait, C. Tsui, M. A. van Zeeland, J. C. Wesley, and J. H. Yu, Nucl. Fusion 53, 083004 (2013).

${ }^{68}$ T. Fülöp, G. Pokol, P. Helander, and M. Lisak, Phys. Plasmas 13, 062506 (2006).

${ }^{69}$ V. Kiptily and JET-EFDAT Contributors, "Runaway measurements on JET," Joint meeting of ITPA MHD and EP TGs, Abingdon, United Kingdom (2013); A. E. Shevelev, E. M. Khilkevitch, V. G. Kiptily, I. N. Chugunov, D. B. Gin, D. N. Doinikov, V. O. Naidenov, A. E. Litvinov, I. A. Plunovskii, and JET-EFDA Contributors, Nucl. Fusion 53, 123004 (2013).

${ }^{70} \mathrm{~T}$. Kudyakov, "Spectral measurements of runaway electrons in the TEXTOR tokamak," Ph.D. dissertation (University of Düsseldorf, 2009).

${ }^{71}$ M. Forster, K. H. Finken, M. Lehnen, J. Linke, B. Schweer, C. Thomser, O. Willi, Y. Xu, and TEXTOR Team, Nucl. Fusion 51, 043003 (2011).

${ }^{72}$ M. Forster, K. H. Finken, M. Lehnen, O. Willi, Y. Xu, and TEXTOR Team, Phys. Plasmas 19, 052506 (2012).

${ }^{73}$ R. Jaspers, N. J. Lopes Cardozo, A. J. H. Donne, H. L. M. Widdershoven, and K. H. Finken, Rev. Sci. Instrum. 72, 466 (2001).

${ }^{74}$ J. H. Yu, E. M. Hollmann, N. Commaux, N. W. Eidietis, D. A. Humphreys, A. N. James, T. C. Jernigan, and R. A. Moyer, Phys. Plasmas 20, 042113 (2013).

${ }^{75}$ M. Lehnen, S. S. Abdullaev, G. Arnoux, S. A. Bozhenkov, M. W. Jakubowski, R. Jaspers, V. V. Plyusnin, V. Riccardo, U. Samm, JE EFDA Contributors, and TEXTOR Team, J. Nucl. Mater. 390, 740 (2009).

${ }^{76}$ G. Maddaluno, G. Maruccia, M. Merola, and S. Rollet, J. Nucl. Mater. 313, 651 (2003).

${ }^{77}$ B. Bazylev, G. Arnoux, W. Fundamenski, Yu. Igitkhanov, M. Lehnen, and JET EFDA Contributors, J. Nucl. Mater. 415, S841 (2011).

${ }^{78}$ V. Sizyuk and A. Hassanein, Nucl. Fusion 49, 095003 (2009).

${ }^{79}$ J. Riemann, H. M. Smith, and P. Helander, Phys. Plasmas 19, 012507 (2012).

${ }^{80}$ J. R. Martin-Solis, A. Loarte, E. M. Hollmann, B. Esposito, V. Riccardo, FTU, DIII-D Teams and JET EFDA Contributors, Nucl. Fusion 54, 083027 (2014).

${ }^{81}$ M. Lehnen, S. A. Bozhenkov, S. S. Abdullaev, M. W. Jakubowski, and TEXTOR Team, Phys. Rev. Lett. 100, 255003 (2008).
${ }^{82}$ N. Commaux, L. R. Baylor, S. K. Combs, N. W. Eidietis, T. E. Evans, C. R. Foust, E. M. Hollmann, D. A. Humphreys, V. A. Izzo, A. N. James, T. C. Jernigan, S. J. Meitner, P. B. Parks, J. C. Wesley, and J. H. Yu, Nucl. Fusion 51, 103001 (2011).

${ }^{83}$ P. B. Aleynikov, A. A. Ivanov, R. R. Khayrutdinov, S. V. Konovalov, V. E. Lukash, S. Yu. Medvedev, and S. Putvinski, "Simulations of runaway electron transport under MHD perturbations in ITER," in 37th EPS Conference on Plasma Physics (2010), p. P1.1004.

${ }^{84}$ G. Papp, M. Drevlak, T. Fülöp, and G. I. Pokol, Plasma Phys. Controlled Fusion 54, 125008 (2012).

${ }^{85}$ V. E. Lukash, A. A. Kavin, Yu. V. Gribov, R. R. Khayrutdinov, and A. Loarte, "Study of ITER plasma position control during disruptions with formation of runaway electrons," paper presented at ITPA MHD Stability Topical Group (2013).

${ }^{86}$ S. Putvinski, L. Baylor, D. Campbell, V. Chuyanov, Yu. Gribov, V. Leonov, A. Loarte, S. Maruyama, R. Pearce, R. A. Pitts, A. Polevoi, R. Mitteau, and M. Sugihara, "Disruption mitigation in ITER," in IAEA Fusion Energy Conference (2010), poster No. ITER/1-6.

${ }^{87}$ P. B. Parks and W. Wu, Nucl. Fusion 54, 023002 (2014)

${ }^{88}$ T. Kudyakov, K. H. Finken, M. W. Jakubowski, M. Lehnen, Y. Xu, B. Schweer, G. van Wassenhove, O. Willi, and TEXTOR Team, Nucl. Fusion 48, 122002 (2008).

${ }^{89}$ V. A. Izzo, A. N. James, E. M. Hollmann, J. H. Yu, D. A. Humphreys, J. C. Wesley, L. L. Lao, P. B. Parks, P. E. Sieck, D. G. Whyte, G. J. Olynyk, and R. S. Granetz, "Runaway electron confinement and modeling for DIII-D, Alacator C-MOD, and ITER," in IAEA Fusion Energy Conference (2010), poster No. THS/P9-2.

${ }^{90}$ N. W. Eidietis, N. Commaux, E. M. Hollmann, D. A. Humphreys, T. C. Jernigan, R. A. Moyer, E. J. Strait, M. A. van Zeeland, J. C. Wesley, and J. H. Yu, Phys. Plasmas 19, 056109 (2012).

${ }^{91}$ F. Saint-Laurent, C. Reux, J. Bucalossi, A. Loarte, S. Bremond, C. Gil, P. Maget, Ph. Moreau, and J. L. Segui, "Disruptions and runaways electron mitigations studies on Tore Supra," in IAEA Fusion Energy Conference (2010), poster No. EXS//P2-16.

${ }^{92}$ E. M. Hollmann, T. C. Jernigan, M. Groth, D. G. Whyte, D. S. Gray, M. E. Austin, B. D. Bray, D. P. Brennan, N. H. Brooks, T. E. Evans, D. A. Humphreys, C. J. Lasnier, R. A. Moyer, A. G. McLean, P. B. Parks, V. Rozhansky, D. L. Rukdakov, E. J. Strait, and W. P. West, Nucl. Fusion 45, 1046 (2005).

${ }^{93}$ M. L. Reinke, D. G. Whyte, R. Granetz, and I. H. Hutchinson, Nucl. Fusion 48, 125004 (2008). 